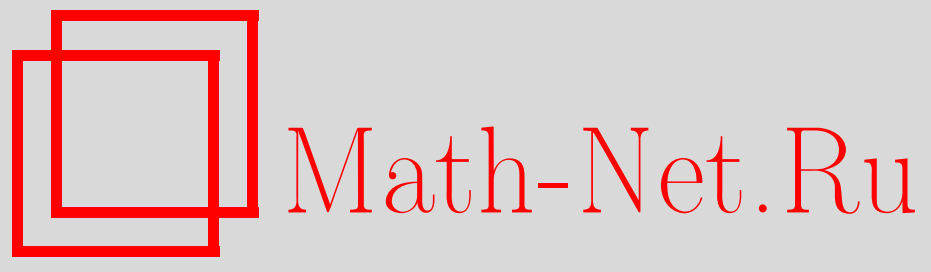

Г. Е. Иванов, Теорема о седловой точке для сильно и слабо выпуклых функций, Изв. РАН. Сер. матем., 2011, том 75, выпуск 1, 71-100

DOI: https://doi.org/10.4213/im4103

Использование Общероссийского математического портала Math-Net.Ru подразумевает, что вы прочитали и согласны с пользовательским соглашением http://www . mathnet.ru/rus/agreement

Параметры загрузки:

IP: 54.166 .219 .16

26 апреля 2023 г., 18:08:31

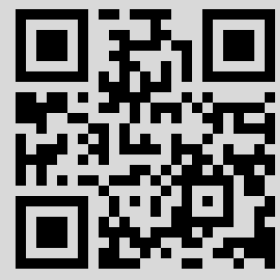




\title{
Г. Е. Иванов \\ Теорема о седловой точке для сильно и слабо выпуклых функций
}

\begin{abstract}
Доказана теорема о существовании, единственности и непрерывной зависимости от параметров седловой точки в минимаксной задаче, возникающей, например, в теории дифференциальных игр. Полученная теорема существования седловой точки не следует из известных теорем Дж. фон Неймана, Ки Фаня, М. Сайона и других теорем, так как пересечение множеств подуровней рассматриваемой функции может оказаться не связным и не пустым. Условия полученной теоремы сформулированы в терминах свойств сильной выпуклости и слабой выпуклости функций, заданных в банаховом пространстве. Исследованы свойства сильно и слабо выпуклых функций, связанные с операциями минимизации и максимизации. Получены неулучшаемые оценки параметров выпуклости для инфимальной конволюции (эписуммы) и эпиразности функций, в результате чего построено исчисление параметров выпуклости функций относительно эпиопераций. Приведены характерные примеры и показана существенность предположений доказанных теорем.
\end{abstract}

Библиография: 11 наименований.

Ключевые слова: седловая точка, минимакс, сильная выпуклость и слабая выпуклость, дифференциальная игра.

\section{Введение}

В 11 настоящей статьи даны определения, необходимые для формулировки теоремы о седловой точке, сформулирована эта теорема и приведен пример, показывающий, что в принятых предположениях пересечение множеств подуровней целевой функции может быть не связным. В 22 введены обозначения, используемые в дальнейшем изложении, получены свойства и приведены характерные примеры сильно и слабо выпуклых функций, показана существенность предположений, принятых в теореме о седловой точке. В 33 получены вспомогательные результаты и теорема об исчислении параметров выпуклости. В $\S 4$ теорема о седловой точке доказана. В $\S 5$ доказана непрерывная зависимость седловой точки от параметров. В $\S 6$ рассмотрено приложение полученной теоремы о седловой точке в теории дифференциальных игр.

Работа выполнена при финансовой поддержке РФФИ (грант №10-01-00139а) и ФЦП "Научные и научно-педагогические кадры инновационной России" (контракты П 938 и 16.740.11.0128). 


\section{§ 1. Формулировка теоремы о седловой точке}

Пусть $E$ - банахово пространство. Обозначим $\overline{\mathbb{R}}=\mathbb{R} \cup\{-\infty,+\infty\}$. Напомним, что надграфиком функции $f: E \rightarrow \overline{\mathbb{R}}$ называется множество ері $f=$ $\{(x, y) \in E \times \mathbb{R}: x \in E, y \geqslant f(x)\} ;$ эффективным множеством этой функции называется множество $\operatorname{dom} f=\{x \in E: f(x) \in \mathbb{R}\}$.

Функция $w: E \rightarrow \overline{\mathbb{R}}$ называется коэриитивной, если

$$
\lim _{\|x\| \rightarrow \infty} \frac{w(x)}{\|x\|}=+\infty .
$$

Функция $w: E \rightarrow \mathbb{R}$ называется равномерно въпуклой на выпуклом множестве $B \subset E$, если

$$
\inf _{\substack{x_{1}, x_{2} \in B \\\left\|x_{1}-x_{2}\right\| \geqslant \varepsilon}}\left(\lambda w\left(x_{1}\right)+(1-\lambda) w\left(x_{2}\right)-w\left(\lambda x_{1}+(1-\lambda) x_{2}\right)\right)>0 \quad \forall \lambda \in(0,1), \quad \forall \varepsilon>0 .
$$

Пусть $w: E \rightarrow \mathbb{R} \cup\{+\infty\}$ - выпуклая полунепрерывная снизу функция, $\operatorname{dom} w \neq \varnothing$. Выпуклая полунепрерывная снизу функция $f: E \rightarrow \mathbb{R} \cup\{+\infty\}$ называется силъно выпуклой относительно функции $w$, если существует функция $g: E \rightarrow \mathbb{R} \cup\{+\infty\}$ такая, что

$$
w(x)=\inf _{u \in E}(f(x-u)+g(u)) \quad \forall x \in E .
$$

Функция $f: E \rightarrow \overline{\mathbb{R}}$ называется слабо выпуклой относительно функции $w$, если $\operatorname{dom} f \neq \varnothing$ и существует функция $g: E \rightarrow \overline{\mathbb{R}}$ такая, что

$$
f(x)=\sup _{u \in \operatorname{dom} w}(g(x+u)-w(u)) \quad \forall x \in E .
$$

Функция $f: E \rightarrow \overline{\mathbb{R}}$ называется слабо вогнутой относительно функции $w$, если функция $x \mapsto-f(-x)$ слабо выпукла относительно функции $w$.

Каждой функции $w: E \rightarrow \overline{\mathbb{R}}$ и каждому числу $t>0$ сопоставим функцию $w_{[t]}: E \rightarrow \overline{\mathbb{R}}$, определяемую формулой

$$
w_{[t]}(x)=t w\left(\frac{x}{t}\right) \quad \forall x \in E .
$$

Заметим, что надграфик функции $w_{[t]}$ получается умножением надграфика функции $w$ на число $t$.

ТЕОРЕма 1.1 (о седловой точке). Пусть функиия $w: E \rightarrow \mathbb{R}$ коэриитивна и для любого шара $B \subset E$ функиия $w$ ограничена и равномерно выпукла на этом шаре. Пусть задань числа $r>0, t>0, R>r$ u $T>t$. Пусть функиия $f: E \rightarrow \mathbb{R}$ слабо выпукла относительно функиии $w_{[R]}$ и слабо вогнута относительно функиии $w_{[T]}$, функция $g: E \rightarrow \mathbb{R} \cup\{+\infty\}$ сильно выпукла относительно функиии $w_{[r]}$ и функиия $h: E \rightarrow \mathbb{R} \cup\{+\infty\}$ силъно выпукла относительно функции $w_{[t]}$. Тогда для любого вектора $x \in E$ существует и единственна седловая точка $\left(u_{*}, v_{*}\right) \in E \times E$ функиии

$$
F(u, v)=f(x-u+v)+g(u)-h(v), \quad u, v \in E,
$$


mo ecmb

$$
F\left(u_{*}, v\right)<F\left(u_{*}, v_{*}\right)<F\left(u, v_{*}\right) \quad \forall u, v \in E: u \neq u_{*}, v \neq v_{*} .
$$

ЗАмЕчАниЕ 1.1. Минимаксная задача для функции вида (1.1) возникает, например, в теории игр. При этом векторы $u$ и $v$ - это управления игроков, вектор $x$ - исходное состояние системы, вектор $x-u+v$ - ее конечное состояние, функция $f$ определяет зависимость результата игры от конечного состояния системы, функция $g$ определяет затраты на управление игрока $u$, функция $h-$ затраты на управление игрока $v$.

ЗАмЕчАниЕ 1.2. В теореме 5.1 доказана непрерывная зависимость седловой точки функции (1.1) относительно вектора $x$ в смысле сильной топологии пространства $E$ и относительно функций $f, g, h$ в смысле некоторой специальной топологии, более слабой, чем топология равномерной сходимости.

ПрЕДЛОЖЕНИЕ 1.1. Пусть $E$ - гилъбертово пространство, $C>0 u w(x)=$ $\frac{C}{2}\|x\|^{2}$ для любого $x \in E$. Пусть задана полунепрерьвная снизу функиия $f: E \rightarrow \mathbb{R} \cup\{+\infty\}, \operatorname{dom} f \neq \varnothing$. Тогда:

(i) функиия $f$ сильно выпукла относительно функции $w$, если и только если функиия $x \mapsto f(x)-w(x)$ выпукла;

(ii) функция $f$ слабо выпукла относительно функиии $w$, если и только если функция $x \mapsto f(x)+w(x)$ выпукла.

ДокАзАтельство. Утверждение (i) вытекает из [1, лемма 4.8.1, теорема 4.8.5]. Утверждение (ii) следует из [2, теорема 2.4.2].

В работе [3] введено следующее понятие сильно выпуклой функции. Функция $f: \mathbb{R}^{n} \rightarrow \mathbb{R} \cup\{+\infty\}$ называется сильно выпуклой с константой $C>0$, если функция $x \mapsto f(x)-\frac{C}{2}\|x\|^{2}$ выпукла. Согласно предложению 1.1 рассматриваемые в настоящей работе понятия сильно и слабо выпуклых функций являются обобщениями понятия сильно выпуклой функции, введенного в [3].

ЗАмЕЧАнИЕ 1.3. Если пространство $E$ гильбертово и $w(x)=\|x\|^{2}$, то теорема 1.1 следует из фундаментальной теоремы Дж. фон Неймана [4] и предложения 1.1. Однако в общем случае теорема 1.1 не следует из теоремы Дж. фон Неймана или других известных ее обобщений (см., например, [5], [6]). В этих и многих других теоремах для существования седловой точки функции $F(u, v)$ требуется, чтобы для любого числа $C \in \mathbb{R}$ и любого набора векторов $\left\{v_{1}, \ldots, v_{n}\right\} \subset E$ пересечение множеств подуровней

$$
\bigcap_{k=1}^{n}\left\{u \in E: F\left(u, v_{k}\right) \leqslant C\right\}
$$

было связно или пусто. Следующий пример показывает, что в предположениях теоремы 1.1 последнее условие может не выполняться. 
ПримеР 1.1. Пусть

$$
\begin{gathered}
E=\mathbb{R}^{2}, \quad w\left(x_{1}, x_{2}\right)=\left(x_{1}^{2}+x_{2}^{2}\right)^{2}, \quad f=-w, \\
t=\frac{1}{\sqrt[3]{2}}, \quad g=h=w_{[t]}, \quad C=-4, \\
\bar{x}=(0,0), \quad n=2, \quad \bar{v}_{1}=(0,1), \quad \bar{v}_{2}=(0,-1), \\
F(\bar{u}, \bar{v})=f(\bar{x}-\bar{u}+\bar{v})+g(\bar{u})-h(\bar{v}) .
\end{gathered}
$$

Тогда

$$
\begin{aligned}
& F\left(\bar{u}, \bar{v}_{1}\right)=2\left(u_{1}^{2}+u_{2}^{2}\right)^{2}-\left(u_{1}^{2}+\left(u_{2}-1\right)^{2}\right)^{2}-2, \\
& F\left(\bar{u}, \bar{v}_{2}\right)=2\left(u_{1}^{2}+u_{2}^{2}\right)^{2}-\left(u_{1}^{2}+\left(u_{2}+1\right)^{2}\right)^{2}-2,
\end{aligned}
$$

где $\bar{u}=\left(u_{1}, u_{2}\right)$. Обозначим $U_{k}=\left\{\bar{u}=\left(u_{1}, u_{2}\right) \in \mathbb{R}^{2}: F\left(\bar{u}, \bar{v}_{k}\right) \leqslant C\right\}, k=1,2$. Покажем, что

$$
u_{2} \leqslant 0 \quad \forall\left(u_{1}, u_{2}\right) \in U_{1} .
$$

Предположим противное: существует точка $\bar{u}^{0}=\left(u_{1}^{0}, u_{2}^{0}\right) \in U_{1}$ такая, что $u_{2}^{0}>0$. Поскольку

$\min _{u_{1} \in \mathbb{R}}\left(2\left(u_{1}^{2}+u_{2}^{2}\right)^{2}-\left(u_{1}^{2}+\left(u_{2}-1\right)^{2}\right)^{2}\right)= \begin{cases}-2\left(1-2 u_{2}\right)^{2}, & u_{2} \in(-\sqrt{2}-1, \sqrt{2}-1), \\ 2 u_{2}^{4}-\left(1-u_{2}\right)^{4}, & u_{2} \notin(-\sqrt{2}-1, \sqrt{2}-1),\end{cases}$

то, учитывая неравенство $u_{2}^{0}>0$, получаем

$$
\min _{u_{1} \in \mathbb{R}}\left(2\left(u_{1}^{2}+\left(u_{2}^{0}\right)^{2}\right)^{2}-\left(u_{1}^{2}+\left(u_{2}^{0}-1\right)^{2}\right)^{2}\right)>-2,
$$

поэтому

$$
F\left(\bar{u}^{0}, \bar{v}_{1}\right)=2\left(\left(u_{1}^{0}\right)^{2}+\left(u_{2}^{0}\right)^{2}\right)^{2}-\left(\left(u_{1}^{0}\right)^{2}+\left(u_{2}^{0}-1\right)^{2}\right)^{2}-2>-4=C,
$$

что противоречит включению $\bar{u}^{0} \in U_{1}$. Полученное противоречие доказывает соотношение (1.2). Аналогично, $u_{2} \geqslant 0$ для любой точки $\left(u_{1}, u_{2}\right) \in U_{2}$. Следовательно,

$$
U_{1} \cap U_{2} \subset\left\{\left(u_{1}, 0\right): u_{1} \in \mathbb{R}\right\}
$$

Поэтому

$$
U_{1} \cap U_{2}=\left\{\left(u_{1}, 0\right): 2 u_{1}^{4}-\left(u_{1}^{2}+1\right)^{2} \leqslant-2\right\}=\{(1,0) ;(-1,0)\} .
$$

Таким образом, множество $U_{1} \cap U_{2}$ не пусто и не связно.

\section{§ 2. Свойства сильно и слабо выпуклых функций}

Через $\mathfrak{C}(E)$ обозначим множество выпуклых полунепрерывных снизу функций $w: E \rightarrow \mathbb{R} \cup\{+\infty\}$ таких, что $\operatorname{dom} w \neq \varnothing$. Для заданной функции $w \in \mathfrak{C}(E)$ через $\operatorname{SConv}(w), \operatorname{WConv}(w), \operatorname{WConc}(w)$ обозначим соответственно классы сильно выпуклых, слабо выпуклых и слабо вогнутых функций относительно функции $w$.

Через $\mathfrak{C} \mathfrak{B}(E)$ обозначим множество коэрцитивных функций $w: E \rightarrow \mathbb{R}$ таких, что для любого шара $B \subset E$ каждая функция $w \in \mathfrak{C} \mathfrak{B}(E)$ ограничена и равномерно выпукла на $B$. 
ЗАмЕчаниЕ 2.1. Если существует функция $w \in \mathfrak{C} \mathfrak{B}(E)$, то для любого числа $C>\inf _{x \in E} w(x)$ множество $\{x \in E: w(x)<C\}$ открыто, ограничено и равномерно выпукло. Отсюда следует, что в пространстве $E$ можно ввести равномерно выпуклую норму, эквивалентную исходной. Следовательно, пространство $E$ рефлексивно.

Через $\mathfrak{B}_{R}$ обозначим замкнутый шар радиуса $R$ с центром в нуле: $\mathfrak{B}_{R}=$ $\{x \in E:\|x\| \leqslant R\}$.

ЗАмечАниЕ 2.2. Легко видеть, что если функция $f: \mathfrak{B}_{R} \rightarrow \mathbb{R}$ выпукла и ограничена, то она удовлетворяет условию Липшица на шаре $\mathfrak{B}_{r}$ при $r<R$. Отсюда следует, что любая функция $g \in \mathfrak{C} \mathfrak{B}(E)$ удовлетворяет условию Липшица на любом ограниченном множестве.

Для любых функций $f, g: E \rightarrow \overline{\mathbb{R}}$ рассмотрим эписумму, или, что то же самое, инфимальную конволюиию

$$
(f \boxplus g)(x)=\inf _{u \in E:\left\{\begin{array}{l}
f(x-u)<+\infty \\
g(u)<+\infty
\end{array}\right.}(f(x-u)+g(u)),
$$

и эпиразность

$$
(f \boxminus g)(x)=\sup _{u \in E:\left\{\begin{array}{l}
f(x+u)>-\infty \\
g(u)<+\infty
\end{array}\right.}(f(x+u)-g(u)) .
$$

Инфимальную конволюцию ввел Дж. Моро в работе [7]. Эпиразность предложил рассматривать Е. С. Половинкин в работе [8].

ЗАмЕчАниЕ 2.3. Эписумму и эпиразность функций можно представить соответственно через сумму и разность Минковского надграфиков (эпиграфов) этих функций $[8$, предложения $10.1,10.2]$. С этим обстоятельством связано название этих операций.

Условимся, что в выражениях, не содержащих скобок, операции эписложения и эпивычитания выполняются слева направо.

Непосредственно из определений получаем следующие свойства эпиопераций:

(i) $f \boxplus g=g \boxplus f$;

(ii) $f \boxplus(g \boxplus h)=f \boxplus g \boxplus h$;

(iii) $f \boxminus(g \boxplus h)=f \boxminus g \boxminus h$;

(iv) $f \boxplus g \boxminus g \leqslant f \leqslant f \boxminus g \boxplus g$;

(v) $f \boxplus g \boxminus h \leqslant f \boxminus h \boxplus g$;

(vi) если $f_{1} \leqslant f_{2}, g_{1} \leqslant g_{2}$, то $f_{1} \boxplus g_{1} \leqslant f_{2} \boxplus g_{2}, f_{1} \boxminus g_{2} \leqslant f_{2} \boxminus g_{1}$;

(vii) $f \boxminus g \boxplus g \boxminus g=f \boxminus g, f \boxplus g \boxminus g \boxplus g=f \boxplus g$;

(viii) если $s>0, t>0$, то $\left(f_{[t]}\right)_{[s]}=f_{[t s]},(f \boxplus g)_{[t]}=f_{[t]} \boxplus g_{[t]},(f \boxminus g)_{[t]}=$ $f_{[t]} \boxminus g_{[t]} ;$

(ix) если функция $f$ выпукла, $s>0, t>0$, то $f_{[s]} \boxplus f_{[t]}=f_{[s+t]}$ и $f_{[s+t]} \boxminus$ $f_{[s]}=f_{[t]}$.

Используя свойства (vii) и (iv), получаем следующие критерии сильной и слабой выпуклости. 
Лемма 2.1. Пусть $w \in \mathfrak{C}(E)$. Тогда:

(i) для любой бункиии $f \in \mathfrak{C}(E)$

$$
\begin{aligned}
f \in \operatorname{SConv}(w) & \Leftrightarrow \quad(\exists g: E \rightarrow \mathbb{R} \cup\{+\infty\} \mid f \boxplus g=w) \\
& \Leftrightarrow \quad w \boxminus f \boxplus f=w \quad \Leftrightarrow \quad w \boxminus f \boxplus f \leqslant w ;
\end{aligned}
$$

(ii) для любой функции $f: E \rightarrow \overline{\mathbb{R}}$ такой, что $\operatorname{dom} f \neq \varnothing$, имеем

$$
\begin{aligned}
f \in \operatorname{WConv}(w) & \Leftrightarrow \quad(\exists g: E \rightarrow \overline{\mathbb{R}} \mid g \boxminus w=f) \\
& \Leftrightarrow \quad f \boxplus w \boxminus w=f \quad \Leftrightarrow \quad f \boxplus w \boxminus w \geqslant f ;
\end{aligned}
$$

(iii) для любой функиии $f: E \rightarrow \overline{\mathbb{R}}$ такой, что $\operatorname{dom} f \neq \varnothing$, имеем

$$
\begin{aligned}
f \in \operatorname{WConc}(w) & \Leftrightarrow \quad(\exists g: E \rightarrow \overline{\mathbb{R}} \mid g \boxplus w=f) \\
& \Leftrightarrow \quad f \boxminus w \boxplus w=f \quad \Leftrightarrow \quad f \boxminus w \boxplus w \leqslant f .
\end{aligned}
$$

ЛЕмма 2.2. Для любой функиии $w \in \mathfrak{C}(E)$ справедливо включение

$$
w \in \operatorname{SConv}(w) \cap \operatorname{WConv}(w) \cap \operatorname{WConc}(w) .
$$

ДоказАтельство. Рассмотрим функцию

$$
g(x)= \begin{cases}0, & x=0 \\ +\infty, & x \neq 0\end{cases}
$$

Поскольку $w \boxplus g=w$ и $w_{[2]} \boxminus w=w$ согласно свойству (ix), в силу леммы 2.1 получаем доказываемое включение.

Из леммы 2.1 вытекают следующие две леммы.

Лемма 2.3. Пусть $w \in \mathfrak{C}(E), g \in \operatorname{SConv}(w)$. Тогда

$$
\begin{gathered}
\operatorname{SConv}(g) \subset \operatorname{SConv}(w), \quad \operatorname{WConv}(w) \subset \operatorname{WConv}(g), \\
\operatorname{WConc}(w) \subset \operatorname{WConc}(g) .
\end{gathered}
$$

Лемма 2.4. Пусть заданы функции $w \in \mathfrak{C}(E)$ u $f, h: E \rightarrow \overline{\mathbb{R}}$. Тогда:

(i) еслu $f \in \operatorname{WConv}(w), \operatorname{dom}(f \boxminus h) \neq \varnothing$, mo $f \boxminus h \in \operatorname{WConv}(w)$;

(ii) ecлu $f \in \operatorname{WConc}(w), \operatorname{dom}(f \boxplus h) \neq \varnothing$, mo $f \boxplus h \in \operatorname{WConc}(w)$.

Лемма 2.5. Пусть задань числа $T>t>0$ и функиия $w \in \mathfrak{C}(E)$. Тогда

$$
\begin{gathered}
\operatorname{SConv}\left(w_{[t]}\right) \subset \operatorname{SConv}\left(w_{[T]}\right), \quad \operatorname{WConv}\left(w_{[T]}\right) \subset \operatorname{WConv}\left(w_{[t]}\right), \\
\operatorname{WConc}\left(w_{[T]}\right) \subset \operatorname{WConc}\left(w_{[t]}\right) .
\end{gathered}
$$

ДокАЗАТЕльство. В силу свойства (iх) эпиопераций справедливо включение $w_{[t]} \in \operatorname{SConv}\left(w_{[T]}\right)$. Применяя лемму 2.3, получаем доказываемые включения.

Лемма 2.6. Пусть заданъц число $t>0$ и функиия $w \in \mathfrak{C}(E)$. Тогда:

(i) если $f \in \operatorname{SConv}(w)$, mo $f_{[t]} \in \operatorname{SConv}\left(w_{[t]}\right)$;

(ii) ecлu $f \in \operatorname{WConv}(w)$, mo $f_{[t]} \in \operatorname{WConv}\left(w_{[t]}\right)$;

(iii) если $f \in \operatorname{WConc}(w)$, mo $f_{[t]} \in \operatorname{WConc}\left(w_{[t]}\right)$. 
ДоКАЗАТЕЛЬСтво состоит в применении леммы 2.1 и свойства (viii) эпиопераций.

Известно [2, теорема 2.3.1, (5)], что надграфик эписуммы двух функций с точностью до замыкания совпадает с суммой Минковского надграфиков этих функций. Поэтому согласно лемме 2.1 сильная выпуклость функции $f$ относительно функции $w$ означает, что надграфик функции $f$ является слагаемым (в смысле суммы Минковского) надграфика функции $w$.

Приведем пример сильно выпуклых функций в произвольном банаховом пространстве.

ПримеР 2.1. Пусть $\varphi: \mathbb{R} \rightarrow \mathbb{R}$ - произвольная четная выпуклая функция, $E$ - произвольное банахово пространство, $\alpha>0$,

$$
\begin{gathered}
w(x)=\varphi(\|x\|), \quad f(x)= \begin{cases}\varphi(\|x\|), & \|x\| \leqslant \alpha, \\
+\infty, & \|x\|>\alpha,\end{cases} \\
g(x)=\varphi(\|x\|+\alpha)-\varphi(\alpha) \quad \forall x \in E .
\end{gathered}
$$

Тогда $f \boxplus g=w$. Поэтому функции $f$ и $g$ сильно выпуклы относительно функции $w$.

Из теоремы отделимости вытекает следующая лемма.

ЛЕмма 2.7. Пусть заданъ функции $f, w \in \mathfrak{C}(E)$ и функиия w коэриитивна. Тогда $f \in \operatorname{WConv}(w)$.

Лемма 2.8. Пусть $w \in \mathfrak{C}(E)$. Для функиии $f: E \rightarrow \mathbb{R}$ включение $f \in$ $\mathrm{WConv}(w)$ эквивалентно условию

$\forall x \in E, \quad \forall \varepsilon>0 \quad \exists y \in \operatorname{dom} w: \quad \forall u \in E \quad f(x+u)-f(x) \geqslant-w(y-u)+w(y)-\varepsilon$.

ДокАзАтЕльство. В силу определения эписуммы условие (2.1) эквивалентно соотношению

$$
\forall x \in E, \quad \forall \varepsilon>0 \quad \exists y \in \operatorname{dom} w: \quad f(x)+w(y) \leqslant(f \boxplus w)(x+y)+\varepsilon,
$$

то есть

$$
\forall x \in E \quad f(x) \leqslant \sup _{y \in \operatorname{dom} w}((f \boxplus w)(x+y)-w(y))=(f \boxplus w \boxminus w)(x) .
$$

Применяя лемму 2.1,(ii), получаем доказываемое утверждение.

Лемма 2.8 показывает, что геометрически слабая выпуклость функции $f$ относительно функции $w$ означает возможность "подстыковать" снизу к любой точке графика функции $f$ сдвиг графика функции $x \mapsto-w(-x)$ с любой заданной точностью $\varepsilon$.

ЗАМЕЧАНИЕ 2.4. Из леммы 2.8 следует, что если заданы функции $w \in \mathfrak{C}(E)$, $f: E \rightarrow \mathbb{R}$ такие, что

$$
\forall x \in E \quad \exists y \in \operatorname{dom} w: \forall u \in E \quad f(x+u)-f(x) \geqslant-w(y-u)+w(y),
$$


то $f \in \operatorname{WConv}(w)$. Соотношение (2.2) можно переписать в виде

$$
\forall x \in E \quad \exists y \in \operatorname{dom} w:(f \boxplus w)(x+y)=f(x)+w(y) .
$$

Из условия $f \in \operatorname{WConv}(w)$, вообще говоря, не следует соотношение (2.2). Например, если $E=\mathbb{R}, f(x)=x, w(x)=\sqrt{1+x^{2}}$, то $f \in \operatorname{WConv}(w)$, однако соотношение (2.2) не выполнено.

В теореме 1.1 предполагается, что функция $f$ одновременно слабо выпукла относительно функции $w_{[R]}$ и слабо вогнута относительно функции $w_{[T]}$. При этом согласно лемме 2.5 функция $f$ будет слабо выпукла и слабо вогнута относительно функции $w_{[\min \{R, T\}]}$. Предложение 2.1 дает характеризацию класса функций, одновременно слабо выпуклых и слабо вогнутых относительно функции $w(x)=\frac{C}{2}\|x\|^{2}$ в гильбертовом пространстве.

ПреДЛОжЕНИЕ 2.1 [2, теорема 2.1.2]. Пусть $E$ - гилъбертово пространство, и пусть $C>0$ и $w(x)=\frac{C}{2}\|x\|^{2}$ для любого $x \in E$. Функиия $f: E \rightarrow \mathbb{R}$ принадлежит множеству $\operatorname{WConv}(w) \cap \operatorname{WConc}(w)$ тогда и только тогда, когда функиия $f: E \rightarrow \mathbb{R}$ дифферениируема и производная Фреше функции $f$ удовлетворяет условию Липшича с константой $C$.

Если функция $f$ сильно выпукла (или слабо выпукла, или слабо вогнута) относительно функции $w_{[t]}$, то, учитывая лемму 2.5 , число $t$ можно назвать параметром сильной выпуклости (или слабой выпуклости, или слабой вогнутости) функции $f$ относительно функции $w$. Следующая лемма показывает, что любой многочлен четвертой степени является с некоторыми параметрами, зависящими от этого многочлена, одновременно слабо выпуклой и слабо вогнутой функцией относительно функции $w(x)=x^{4}+2 x^{2}$.

Лемма 2.9. Пусть $w(x)=x^{4}+2 x^{2}, f(x)$ - произвольный многочлен четвертой степени, $x \in \mathbb{R}$. Тогда существуют числа $s, t>0$ такие, что $f \in \operatorname{WConv}\left(w_{[s]}\right) \cap \operatorname{WConc}\left(w_{[t]}\right)$.

ДокАЗАТЕЛЬСтво. Пусть $f(x)=a_{4} x^{4}+a_{3} x^{3}+a_{2} x^{2}+a_{1} x+a_{0}, a_{4} \neq 0$. Поскольку $\lim _{x \rightarrow \infty}\left(2\left|a_{4} x^{3}\right|-\left|3 a_{3} x^{2}+2 a_{2} x+a_{1}\right|\right)=+\infty$, существует число $x_{0}>0$ такое, что

$$
2\left|a_{4} x^{3}\right| \geqslant\left|3 a_{3} x^{2}+2 a_{2} x+a_{1}\right|+8 \quad \forall x \in \mathbb{R}:|x| \geqslant x_{0} .
$$

Определим число $C>0$ так, что

$$
a_{4}\left(u^{2}+4 x u+6 x^{2}\right)+a_{3}(u+3 x)+a_{2} \leqslant C\left(u^{2}+x^{2}+1\right) \quad \forall x \in \mathbb{R}, \quad \forall u \in \mathbb{R} .
$$

Определим числа

$$
\tau=\max \left\{(5 C)^{1 / 3}, C\left(x_{0}^{2}+1\right), 4 C a_{4}^{-2 / 3}\right\}, \quad t=\frac{1}{\tau} .
$$

Зафиксируем произвольное число $x$. Определим число $y$ из равенства

$$
f^{\prime}(x)=w_{[t]}^{\prime}(y)
$$


Поскольку $w_{[t]}(y)=\tau^{3} y^{4}+2 \tau y^{2}, w_{[t]}^{\prime}(y)=4\left(\tau^{3} y^{3}+\tau y\right), w_{[t]}^{\prime \prime}(y) \geqslant 4 \tau>0$, равенство (2.6) определяет единственное число $y$. Покажем, что

$$
C\left(u^{2}+x^{2}+1\right) \leqslant \tau^{3}\left(\frac{u^{2}}{5}+y^{2}\right)+2 \tau \quad \forall u \in \mathbb{R} .
$$

Если $|x| \leqslant x_{0}$, то в силу первого из равенств $(2.5)$ имеем $C \leqslant \frac{\tau^{3}}{5}$ и $C\left(x^{2}+1\right) \leqslant 2 \tau$, что доказывает неравенство (2.7) в этом случае. Рассмотрим случай $|x|>x_{0}$. Из равенства (2.6) получаем

$$
4 a_{4} x^{3}+3 a_{3} x^{2}+2 a_{2} x+a_{1}=4 \tau y\left(\tau^{2} y^{2}+1\right) .
$$

В силу соотношения (2.3) имеем $\left|4 a_{4} x^{3}+3 a_{3} x^{2}+2 a_{2} x+a_{1}\right| \geqslant 2\left|a_{4} x^{3}\right| \geqslant 8$. Поэтому $4 \tau|y|\left(\tau^{2} y^{2}+1\right) \geqslant 2\left|a_{4} x^{3}\right| \geqslant 8$. Следовательно, $\tau|y| \geqslant 1$, а значит, $8 \tau^{3}|y|^{3} \geqslant 4 \tau|y|\left(\tau^{2} y^{2}+1\right) \geqslant 2\left|a_{4} x^{3}\right|, \tau^{3} y^{2} \geqslant \frac{\tau}{4} a_{4}^{2 / 3} x^{2}$. Используя первое из равенств (2.5), получаем $C x^{2} \leqslant \tau^{3} y^{2}, C \leqslant \frac{\tau^{3}}{5}$ и $C \leqslant 2 \tau$. Отсюда следует выполнение неравенства (2.7). Из неравенств $(2.4),(2.7)$ получаем

$$
a_{4}\left(u^{2}+4 x u+6 x^{2}\right)+a_{3}(u+3 x)+a_{2} \leqslant \tau^{3}\left(\frac{u^{2}}{5}+y^{2}\right)+2 \tau \quad \forall u \in \mathbb{R} .
$$

Поэтому в силу соотношений $\frac{4}{5} u^{2}+4 y u+5 y^{2}=5\left(y+\frac{2 u}{5}\right)^{2} \geqslant 0$ имеем

$$
a_{4}\left(u^{2}+4 x u+6 x^{2}\right)+a_{3}(u+3 x)+a_{2} \leqslant \tau^{3}\left(u^{2}+4 y u+6 y^{2}\right)+2 \tau \quad \forall u \in \mathbb{R} .
$$

Следовательно,

$$
\begin{aligned}
& a_{4}\left(u^{4}+4 x u^{3}+6 x^{2} u^{2}\right)+a_{3}\left(u^{3}+3 x u^{2}\right)+a_{2} u^{2} \\
& \leqslant \tau^{3}\left(u^{4}+4 y u^{3}+6 y^{2} u^{2}\right)+2 \tau u^{2} \quad \forall u \in \mathbb{R} .
\end{aligned}
$$

Отсюда и из равенства $(2.8)$ для любого $u \in \mathbb{R}$ получаем неравенство

$$
\begin{gathered}
a_{4}\left(u^{4}+4 x u^{3}+6 x^{2} u^{2}+4 x^{3} u\right)+a_{3}\left(u^{3}+3 x u^{2}+3 x^{2} u\right)+a_{2}\left(u^{2}+2 x u\right)+a_{1} u \\
\leqslant \tau^{3}\left(u^{4}+4 y u^{3}+6 y^{2} u^{2}+4 y^{3} u\right)+2 \tau\left(u^{2}+2 y u\right),
\end{gathered}
$$

то есть

$$
f(x+u)-f(x) \leqslant w_{[t]}(y+u)-w_{[t]}(y) \quad \forall u \in \mathbb{R} .
$$

Следовательно,

$$
f(x)-w_{[t]}(y)=\max _{u \in \mathbb{R}}\left(f(x+u)-w_{[t]}(y+u)\right)=\left(f \boxminus w_{[t]}\right)(x-y) .
$$

Поэтому $f(x)=\left(f \boxminus w_{[t]}\right)(x-y)+w_{[t]}(y) \geqslant\left(f \boxminus w_{[t]} \boxplus w_{[t]}\right)(x)$. Отсюда в силу леммы 2.1,(iii) получаем включение $f \in \operatorname{WConc}\left(w_{[t]}\right)$. Существование числа $s>0$ такого, что $f \in \operatorname{WConv}\left(w_{[s]}\right)$, доказывается аналогично. Лемма доказана.

В заключение этого параграфа сделаем несколько замечаний о существенности различных предположений теоремы 1.1. 
ЗАМЕЧАНИЕ 2.5. Предположение о коэрцитивности функции $w$ в теореме 1.1 существенно. Действительно, рассмотрим функции $w(x)=\sqrt{1+x^{2}}$ и $f(x)=x$, заданные на пространстве $E=\mathbb{R}$. Поскольку $f \boxplus w=f \boxminus w=f$, то $f \in \operatorname{WConv}(w) \cap \operatorname{WConc}(w)$. Положим $g=h=w_{[1 / 2]}$. Тогда выполнены все условия теоремы 1.1 , кроме коэрцитивности функции $w$, но для любого $x \in \mathbb{R}$ функция (1.1) не имеет седловой точки.

ЗАмечАниЕ 2.6. Предположение, принятое в теореме 1.1, о том, что функция $w$ равномерно выпукла на любом ограниченном множестве, существенно. Действительно, пусть

$$
\begin{gathered}
E=\mathbb{R}^{2}, \quad B=\left\{\left(x_{1}, x_{2}\right):\left|x_{1}\right|+\left|x_{2}\right| \leqslant 3,\left|x_{1}\right| \leqslant 2\right\}, \\
\widetilde{w}\left(x_{1}, x_{2}\right)= \begin{cases}0, & \left(x_{1}, x_{2}\right) \in B, \\
+\infty, & \left(x_{1}, x_{2}\right) \notin B,\end{cases} \\
\widetilde{\widetilde{w}}\left(x_{1}, x_{2}\right)=\left(1+\sqrt{x_{1}^{2}+x_{2}^{2}}\right)^{2}, \quad w=\widetilde{w} \boxplus \widetilde{\widetilde{w}} \\
s(t)=\left\{\begin{array}{ll}
0, & t \leqslant 0, \\
t, & t \in(0,1), \\
1, & t>1,
\end{array} \quad f\left(x_{1}, x_{2}\right)=s\left(\left|x_{1}\right|-\left|x_{2}\right|+2\right) .\right.
\end{gathered}
$$

Тогда функция $w: \mathbb{R}^{2} \rightarrow \mathbb{R}$ выпукла, коэрцитивна и ограничена на любом ограниченном множестве. Поскольку

$$
\begin{gathered}
(f \boxplus w)\left(x_{1}, x_{2}\right)=(f \boxplus \widetilde{w})\left(x_{1}, x_{2}\right)=s\left(\left|x_{1}\right|-\left|x_{2}\right|-1\right), \\
(f \boxminus w)\left(x_{1}, x_{2}\right)=(f \boxminus \widetilde{w})\left(x_{1}, x_{2}\right)=s\left(\left|x_{1}\right|-\left|x_{2}\right|+5\right), \\
f \boxplus w \boxminus w=f \boxminus w \boxplus w=f,
\end{gathered}
$$

то $f \in \operatorname{WConv}(w) \cap \operatorname{WConc}(w)$. Пусть $g=w_{[2 / 3]}, h=w_{[1 / 3]}$. Тогда согласно лемме 2.2 имеем $g \in \operatorname{SConv}\left(w_{[2 / 3]}\right), h \in \operatorname{SConv}\left(w_{[1 / 3]}\right)$. Прямые вычисления показывают, что

$$
\begin{gathered}
(f \boxminus h \boxplus g)\left(x_{1}, x_{2}\right)=\left(f \boxplus w_{[1 / 3]}\right)\left(x_{1}, x_{2}\right)=s\left(\left|x_{1}\right|-\left|x_{2}\right|+1\right), \\
(f \boxplus g)\left(x_{1}, x_{2}\right)=s\left(\left|x_{1}\right|-\left|x_{2}\right|\right), \\
(f \boxplus g \boxminus h)\left(x_{1}, x_{2}\right)=s\left(\min \left\{\left|x_{1}\right|-\left|x_{2}\right|+1,\left|x_{1}\right|+\frac{2}{3}\right\}\right) .
\end{gathered}
$$

Таким образом,

$$
(f \boxplus g \boxminus h)(0,0)=\frac{2}{3}<1=(f \boxminus h \boxplus g)(0,0) .
$$

Поэтому для функции $F(u, v)=f(v-u)+g(u)-h(v)$ имеет место неравенство

$$
\sup _{v \in E} \inf _{u \in E} F(u, v)<\inf _{u \in E} \sup _{v \in E} F(u, v) .
$$

Следовательно, седловая точка функции $F(u, v)$ не существует. 
ЗАмечание 2.7. Строгие неравенства $R>r, T>t$ в предположениях теоремы 1.1 существенны. Пусть, например, $E=\mathbb{R}$,

$$
w(x)=f(x)=h(x)=x^{2}, \quad g(x)=\left\{\begin{array}{ll}
0, & x=0, \\
+\infty, & x \neq 0,
\end{array} \quad \forall x \in \mathbb{R} .\right.
$$

Тогда $f \in \operatorname{WConv}(w) \cap \operatorname{WConc}(w), g \in \operatorname{SConv}(w), h \in \operatorname{SConv}(w)$. Однако функция

$$
F(u, v)=f(x-u+v)+g(u)-h(v)= \begin{cases}x^{2}+2 x v, & u=0, \\ +\infty, & u \neq 0,\end{cases}
$$

не имеет седловой точки при $x \neq 0$.

\section{§ 3. Вспомогательные результаты}

Через $\langle p, x\rangle$ обозначим значение функционала $p \in E^{*}$ на векторе $x \in E$. Напомним, что субдифференциалом функции $f: E \rightarrow \mathbb{R} \cup\{+\infty\}$ в точке $x_{0} \in$ $\operatorname{dom} f$ называется множество

$$
\partial f\left(x_{0}\right)=\left\{p \in E^{*}: f(x)-f\left(x_{0}\right) \geqslant\left\langle p, x-x_{0}\right\rangle \forall x \in E\right\} .
$$

Непосредственно из определений легко получить следующие леммы.

Лемма 3.1. Для любых функиий $g, h: E \rightarrow \mathbb{R} \cup\{+\infty\}$, векторов $a \in \operatorname{dom} g$, $b \in \operatorname{dom} h$ и функиионала $p \in E^{*}$ следующие условия эквивалентны:

(i) $p \in \partial g(a) \cap \partial h(b)$;

(ii) $(g \boxplus h)(a+b)=g(a)+h(b) u p \in \partial(g \boxplus h)(a+b)$.

Лемма 3.2. Для любъх функиий $f, g, h: E \rightarrow \mathbb{R} \cup\{+\infty\}$ и векторов $x \in E$, $y \in \operatorname{dom}(f \boxminus g)$ справедливо неравенство

$$
(f \boxplus h)(x)-(f \boxminus g)(y) \leqslant(h \boxplus g)(x-y) .
$$

Лемма 3.3. Пусть задань функиии $g, h \in \mathfrak{C}(E)$, одна из которьх коэриитивна. Тогда $g \boxplus h \in \mathfrak{C}(E) u$

$$
\forall x \in \operatorname{dom}(g \boxplus h) \exists y \in E: \quad(g \boxplus h)(x)=g(y)+h(y-x) .
$$

Лемма 3.4. Пусть заданы функиии $g, h \in \mathfrak{C}(E)$. Функиия $g \boxplus h$ коэриитивна тогда и только тогда, когда обе функиии $g$ и $h$ коэриитивны.

Лемма 3.5. Пусть функция $w: E \rightarrow \mathbb{R}$ выпукла, коэрцитивна и ограничена на любом ограниченном множестве; последовательность $\left\{y_{k}\right\} \subset E$ ограничена. Пусть заданы последовательность $\left\{x_{k}\right\} \subset E$ и число $\lambda \in(0,1)$ такие, что последовательность $\left\{w_{[\lambda]}\left(x_{k}\right)-w\left(x_{k}+y_{k}\right)\right\}$ ограничена сверху. Тогда последовательность $\left\{x_{k}\right\}$ ограничена.

ДокАЗАТЕльство. Предположим, что последовательность $\left\{x_{k}\right\}$ неограничена. Выделяя подпоследовательность, будем предполагать, не теряя общности, что $\lim _{k \rightarrow \infty}\left\|x_{k}\right\|=+\infty$ и $\left\|x_{k}\right\|>1$ для любого $k \in \mathbb{N}$. Для любого индекса 
$k \in \mathbb{N}$ определим число $\lambda_{k}=\lambda\left(1-\frac{1}{\left\|x_{k}\right\|}\right)$ и вектор $z_{k}=\frac{1}{1-\lambda_{k}}\left(\frac{x_{k}}{\left\|x_{k}\right\|}+y_{k}\right)$. Поскольку $\lambda_{k} \in(0,1)$ и $x_{k}+y_{k}=\frac{\lambda_{k}}{\lambda} x_{k}+\left(1-\lambda_{k}\right) z_{k}$, то в силу выпуклости функции $w$ имеем

$$
w\left(x_{k}+y_{k}\right) \leqslant \lambda_{k} w\left(\frac{x_{k}}{\lambda}\right)+\left(1-\lambda_{k}\right) w\left(z_{k}\right) \quad \forall k \in \mathbb{N} .
$$

Следовательно,

$$
w_{[\lambda]}\left(x_{k}\right)-w\left(x_{k}+y_{k}\right) \geqslant \frac{\lambda}{\left\|x_{k}\right\|} w\left(\frac{x_{k}}{\lambda}\right)-\left(1-\lambda_{k}\right) w\left(z_{k}\right) \quad \forall k \in \mathbb{N} .
$$

В силу коэрцитивности функции $w$ справедливо соотношение

$$
\lim _{k \rightarrow \infty} \frac{\lambda}{\left\|x_{k}\right\|} w\left(\frac{x_{k}}{\lambda}\right)=+\infty \text {. }
$$

Поскольку последовательность $\left\{z_{k}\right\}$ ограничена, а функция $w$ ограничена на любом ограниченном множестве, последовательность $\left\{\left(1-\lambda_{k}\right) w\left(z_{k}\right)\right\}$ ограничена. Поэтому $\lim _{k \rightarrow \infty}\left(w_{[\lambda]}\left(x_{k}\right)-w\left(x_{k}+y_{k}\right)\right)=+\infty$, что противоречит условиям леммы.

ЛЕмма 3.6. Пусть заданъ положительные числа $\lambda, \mu$, а также функиии $w \in \mathfrak{C} \mathfrak{B}(E), g \in \operatorname{SConv}\left(w_{[\lambda]}\right)$ u векторы $a, b \in E$ maкие, ито $\left(g \boxplus w_{[\mu]}\right)(a+\mu b)=$ $g(a)+w_{[\mu]}(\mu b)$. Тогдa $\left(w_{[\lambda]} \boxminus g\right)(\lambda b-a)=w_{[\lambda]}(\lambda b)-g(a)$.

ДокАЗАТЕльство. Поскольку функция $g \boxplus w_{[\mu]}$ выпукла и ограничена на любом ограниченном множестве, существует функционал $p \in \partial\left(g \boxplus w_{[\mu]}\right)(a+\mu b)$. Отсюда и из равенства $\left(g \boxplus w_{[\mu]}\right)(a+\mu b)=g(a)+w_{[\mu]}(\mu b)$ по лемме 3.1 следует, что $p \in \partial g(a)$ и $p \in \partial w_{[\mu]}(\mu b)=\partial w(b)$. Обозначим $h=w_{[\lambda]} \boxminus g$. Тогда в силу включения $g \in \operatorname{SConv}\left(w_{[\lambda]}\right)$ имеем $w_{[\lambda]}=g \boxplus h$. В силу леммы 3.4 функции $g$ и $h$ коэрцитивны. Согласно лемме 3.3 существует вектор $a_{1} \in E$ такой, что $w_{[\lambda]}(\lambda b)=g\left(a_{1}\right)+h\left(\lambda b-a_{1}\right)$. Отсюда и из включения $p \in \partial w(b)=\partial w_{[\lambda]}(\lambda b)$ в силу леммы 3.1 получаем $p \in \partial g\left(a_{1}\right) \cap \partial h\left(\lambda b-a_{1}\right)$. Поскольку $p \in \partial g(a) \cap$ $\partial h\left(\lambda b-a_{1}\right)$, то, применяя лемму 3.1 в другую сторону, имеем $p \in \partial(g \boxplus h)(a+$ $\left.\lambda b-a_{1}\right)=\partial w_{[\lambda]}\left(a+\lambda b-a_{1}\right)$. Отсюда и из включения $p \in \partial w_{[\lambda]}(\lambda b)$ в силу строгой выпуклости функции $w_{[\lambda]}$ имеем $a=a_{1}$. Следовательно, $w_{[\lambda]}(\lambda b)=$ $g(a)+h(\lambda b-a)=g(a)+\left(w_{[\lambda]} \boxminus g\right)(\lambda b-a)$. Лемма доказана.

Лемма 3.7. Пусть $w \in \mathfrak{C} \mathfrak{B}(E)$, функиия $g \in \mathfrak{C}(E)$ ограничена снизу. Пусть заданы ограниченная последовательность $\left\{x_{k}\right\} \subset E$ и последовательности $\left\{a_{k}\right\} \subset E,\left\{a_{k}^{\prime}\right\} \subset E$ maкuе, что

$$
\max \left\{g\left(x_{k}-a_{k}\right)+w\left(a_{k}\right), g\left(x_{k}-a_{k}^{\prime}\right)+w\left(a_{k}^{\prime}\right)\right\} \leqslant(g \boxplus w)\left(x_{k}\right)+\frac{1}{k} \quad \forall k \in \mathbb{N} .
$$

Тогда $\lim _{k \rightarrow \infty}\left\|a_{k}-a_{k}^{\prime}\right\|=0$.

ДокАЗАтЕЛьство. Зафиксируем вектор $y_{0} \in \operatorname{dom} g$. Поскольку согласно определению эписуммы для любого $k \in \mathbb{N}$ справедливо неравенство $(g \boxplus$ $w)\left(x_{k}\right) \leqslant g\left(y_{0}\right)+w\left(x_{k}-y_{0}\right)$ и функция $w$ ограничена на любом ограниченном множестве, последовательность $\left\{(g \boxplus w)\left(x_{k}\right)\right\}$ ограничена сверху. Отсюда, из 
соотношения (3.1) и ограниченности функции $g$ снизу следует ограниченность сверху последовательностей $\left\{w\left(a_{k}\right)\right\}$ и $\left\{w\left(a_{k}^{\prime}\right)\right\}$. Поэтому, используя коэрцитивность функции $w$, получаем ограниченность последовательностей $\left\{a_{k}\right\}$ и $\left\{a_{k}^{\prime}\right\}$.

В силу выпуклости функции $g$ из соотношения (3.1) следует, что

$$
g\left(x_{k}-\frac{a_{k}+a_{k}^{\prime}}{2}\right)+\frac{w\left(a_{k}\right)+w\left(a_{k}^{\prime}\right)}{2} \leqslant(g \boxplus w)\left(x_{k}\right)+\frac{1}{k} \quad \forall k \in \mathbb{N} .
$$

Поэтому согласно определению эписуммы имеем

$$
\frac{w\left(a_{k}\right)+w\left(a_{k}^{\prime}\right)}{2} \leqslant w\left(\frac{a_{k}+a_{k}^{\prime}}{2}\right)+\frac{1}{k} \quad \forall k \in \mathbb{N} .
$$

Отсюда в силу ограниченности последовательностей $\left\{a_{k}\right\},\left\{a_{k}^{\prime}\right\}$ и равномерной выпуклости функции $w$ на любом ограниченном множестве следует доказываемое утверждение.

Лемма 3.8. Пусть $w \in \mathfrak{C} \mathfrak{B}(E), \mu>0, f \in \operatorname{WConv}(w) \cap \operatorname{WConc}\left(w_{[\mu]}\right), u$ пусть заданы последовательности $\left\{x_{k}\right\} \subset E,\left\{a_{k}\right\} \subset E u\left\{b_{k}\right\} \subset E$ такие, что

$$
\begin{aligned}
f\left(x_{k}\right)+w\left(a_{k}\right) \leqslant(f \boxplus w)\left(x_{k}+a_{k}\right)+\frac{1}{k} & \forall k \in \mathbb{N}, \\
f\left(x_{k}\right)-w_{[\mu]}\left(\mu b_{k}\right) \geqslant\left(f \boxminus w_{[\mu]}\right)\left(x_{k}-\mu b_{k}\right)-\frac{1}{k} & \forall k \in \mathbb{N} .
\end{aligned}
$$

Пусть последовательность $\left\{x_{k}\right\}$ ограничена. Тогда последовательности $\left\{a_{k}\right\}$ $u\left\{b_{k}\right\}$ также ограничены.

Доказательство. Если $\operatorname{dom}(f \boxplus w)=\varnothing$, то $\operatorname{dom}(f \boxplus w \boxminus w)=\varnothing$, что противоречит включению $f \in \operatorname{WConv}(w)$. Следовательно, существует вектор $x_{0} \in \operatorname{dom}(f \boxplus w)$. Поэтому для любого вектора $x \in E$ справедливы неравенства $f(x)+w\left(x_{0}-x\right) \geqslant(f \boxplus w)\left(x_{0}\right)>-\infty$. Отсюда и из включения $w \in \mathfrak{C} \mathfrak{B}(E)$ следует, что функция $f$ ограничена снизу на любом ограниченном множестве. Аналогично, используя включение $f \in \operatorname{WConc}\left(w_{[\mu]}\right)$, получаем ограниченность сверху функции $f$ на любом ограниченном множестве.

Из соотношений $(3.2),(3.3)$ следует, что

$$
\begin{aligned}
& w\left(a_{k}\right)+w_{[\mu]}\left(\mu b_{n}\right)+f\left(x_{k}\right)-f\left(x_{n}\right) \\
& \quad \leqslant(f \boxplus w)\left(x_{k}+a_{k}\right)-\left(f \boxminus w_{[\mu]}\right)\left(x_{n}-\mu b_{n}\right)+\frac{1}{k}+\frac{1}{n} \quad \forall k, n \in \mathbb{N} .
\end{aligned}
$$

Поэтому в силу леммы 3.2 и свойства (ix) эпиопераций имеем

$w\left(a_{k}\right)+w_{[\mu]}\left(\mu b_{n}\right)+f\left(x_{k}\right)-f\left(x_{n}\right) \leqslant w_{[1+\mu]}\left(x_{k}-x_{n}+a_{k}+\mu b_{n}\right)+\frac{1}{k}+\frac{1}{n} \quad \forall k, n \in \mathbb{N}$.

В силу ограниченности последовательности $\left\{x_{k}\right\}$ и ограниченности функции $f$ на любом ограниченном множестве получаем, что последовательности $\left\{w\left(a_{k}\right)-\right.$ $\left.w_{[1+\mu]}\left(x_{k}-x_{1}+a_{k}+\mu b_{1}\right)\right\}$ и $\left\{w_{[\mu]}\left(\mu b_{n}\right)-w_{[1+\mu]}\left(x_{1}-x_{n}+a_{1}+\mu b_{n}\right)\right\}$ ограничены сверху. Применяя лемму 3.5 , получаем требуемое утверждение. 
Лемма 3.9. Пусть $w \in \mathfrak{C} \mathfrak{B}(E), \mu>0, f \in \operatorname{WConv}(w) \cap \operatorname{WConc}\left(w_{[\mu]}\right)$. Тогдa для любого вектора $x_{0} \in E$ существует вектор $\widehat{a}\left(x_{0}\right) \in E$, удовлетворяющий равенствам

$$
\begin{aligned}
(f \boxplus w)\left(x_{0}+\widehat{a}\left(x_{0}\right)\right) & =f\left(x_{0}\right)+w\left(\widehat{a}\left(x_{0}\right)\right), \\
\left(f \boxminus w_{[\mu]}\right)\left(x_{0}-\mu \widehat{a}\left(x_{0}\right)\right) & =f\left(x_{0}\right)-w_{[\mu]}\left(\mu \widehat{a}\left(x_{0}\right)\right) .
\end{aligned}
$$

Кроме того, функция $f$ на любом ограниченном множестве удовлетворяет условию Липшища.

ДокАЗАтЕЛьство. Зафиксируем произвольный вектор $x_{0} \in E$. Поскольку $f \in \operatorname{WConv}(w) \cap \operatorname{WConc}\left(w_{[\mu]}\right)$, то $f \boxplus w \boxminus w=f$ и $f \boxminus w_{[\mu]} \boxplus w_{[\mu]}=f$. Поэтому для любого $k \in \mathbb{N}$ существуют векторы $a_{k}, b_{k} \in E$ такие, что

$$
\begin{gathered}
f\left(x_{0}\right)+w\left(a_{k}\right) \leqslant(f \boxplus w)\left(x_{0}+a_{k}\right)+\frac{1}{k}, \\
f\left(x_{0}\right)-w_{[\mu]}\left(\mu b_{k}\right) \geqslant\left(f \boxminus w_{[\mu]}\right)\left(x_{0}-\mu b_{k}\right)-\frac{1}{k} .
\end{gathered}
$$

Применяя лемму 3.2 и свойство (ix) эпиопераций, получаем

$$
w\left(a_{k}\right)+w_{[\mu]}\left(\mu b_{n}\right) \leqslant w_{[1+\mu]}\left(a_{k}+\mu b_{n}\right)+\frac{1}{k}+\frac{1}{n} \quad \forall k, n \in \mathbb{N} .
$$

Следовательно,

$$
\limsup _{\substack{k \rightarrow \infty \\ n \rightarrow \infty}}\left(w\left(a_{k}\right)+\mu w\left(b_{n}\right)-(1+\mu) w\left(\frac{a_{k}+\mu b_{n}}{1+\mu}\right)\right) \leqslant 0 .
$$

В силу леммы 3.8 последовательности $\left\{a_{k}\right\}$ и $\left\{b_{k}\right\}$ ограничены. Отсюда из равномерной выпуклости функции $w$ на любом ограниченном множестве следует, что

$$
\lim _{\substack{k \rightarrow \infty \\ n \rightarrow \infty}}\left\|a_{k}-b_{n}\right\|=0
$$

Поэтому последовательности $\left\{a_{k}\right\},\left\{b_{n}\right\}$ фундаментальны, а значит, сходятся. В силу соотношения (3.8) пределы этих последовательностей совпадают. Обозначим $\widehat{a}\left(x_{0}\right)=\lim _{k \rightarrow \infty} a_{k}=\lim _{n \rightarrow \infty} b_{n}$.

Из неравенств (3.6), (3.7) имеем

$$
\begin{gathered}
w_{[\mu]}\left(\mu b_{k}\right)-w_{[\mu]}\left(x+\mu b_{k}-x_{0}\right)-\frac{1}{k} \leqslant f\left(x_{0}\right)-f(x) \leqslant w\left(x_{0}+a_{k}-x\right)-w\left(a_{k}\right)+\frac{1}{k} \\
\forall x \in E, \quad \forall k \in \mathbb{N} .
\end{gathered}
$$

Переходя к пределу при $k \rightarrow \infty$ и используя непрерывность функции $w$, получаем

$$
\begin{aligned}
& w_{[\mu]}\left(\mu \widehat{a}\left(x_{0}\right)\right)-w_{[\mu]}\left(x+\mu \widehat{a}\left(x_{0}\right)-x_{0}\right) \leqslant f\left(x_{0}\right)-f(x) \\
& \leqslant w\left(x_{0}+\widehat{a}\left(x_{0}\right)-x\right)-w\left(\widehat{a}\left(x_{0}\right)\right) \quad \forall x \in E .
\end{aligned}
$$

Отсюда по определению эпиопераций получаем, что вектор $\widehat{a}\left(x_{0}\right)$ удовлетворяет равенствам (3.4), (3.5). 
В силу леммы 3.8 функция $\widehat{a}: E \rightarrow E$ ограничена на любом ограниченном множестве. Согласно замечанию 2.2 функция $w$ на любом ограниченном множестве удовлетворяет условию Липшица. Отсюда и из неравенств (3.9) получаем, что функция $f$ на любом ограниченном множестве удовлетворяет условию Липшица. Лемма доказана.

Лемма 3.10. Пусть $w \in \mathfrak{C} \mathfrak{B}(E), \mu>0, f \in \operatorname{WConv}(w) \cap \operatorname{WConc}\left(w_{[\mu]}\right)$, $\lambda \in(0,1), g \in \operatorname{SConv}\left(w_{[\lambda]}\right), y_{0} \in E$. Пусть последовательность $\left\{a_{k}\right\} \subset E$ удовлетворяет соотношению

$$
f\left(y_{0}-a_{k}\right)+g\left(a_{k}\right) \leqslant(f \boxplus g)\left(y_{0}\right)+\frac{1}{k} \quad \forall k \in \mathbb{N} .
$$

Тогда последовательность $\left\{a_{k}\right\}$ сходится.

ДоказАтельство. Поскольку $w_{[\lambda]} \boxminus g \boxplus g=w_{[\lambda]}$, существует вектор $z_{0} \in$ $\operatorname{dom}\left(w_{[\lambda]} \boxminus g\right)$ и выполнено неравенство

$$
w_{[\lambda]}\left(a_{k}+z_{0}\right) \leqslant g\left(a_{k}\right)+\left(w_{[\lambda]} \boxminus g\right)\left(z_{0}\right) \quad \forall k \in \mathbb{N} .
$$

Так как $f \boxplus w \boxminus w=f$, то существует вектор $z_{1} \in \operatorname{dom}(f \boxplus w)$. По определению эписуммы

$$
\begin{gathered}
(f \boxplus w)\left(z_{1}\right) \leqslant f\left(y_{0}-a_{k}\right)+w\left(z_{1}-y_{0}+a_{k}\right) \quad \forall k \in \mathbb{N}, \\
(f \boxplus g)\left(y_{0}\right) \leqslant f\left(y_{0}-a_{1}\right)+g\left(a_{1}\right) \quad \forall k \in \mathbb{N} .
\end{gathered}
$$

Из неравенств (3.10)-(3.13) следует, что

$$
\begin{aligned}
& w_{[\lambda]}\left(a_{k}+z_{0}\right)-w\left(z_{1}-y_{0}+a_{k}\right) \\
& \quad \leqslant\left(w_{[\lambda]} \boxminus g\right)\left(z_{0}\right)-(f \boxplus w)\left(z_{1}\right)+\frac{1}{k}+f\left(y_{0}-a_{1}\right)+g\left(a_{1}\right) \quad \forall k \in \mathbb{N} .
\end{aligned}
$$

Поэтому последовательность $\left\{w_{[\lambda]}\left(a_{k}+z_{0}\right)-w\left(z_{1}-y_{0}+a_{k}\right)\right\}$ ограничена сверху. Следовательно, в силу леммы 3.5 последовательность $\left\{a_{k}\right\}$ ограничена.

В силу леммы 3.9 для любого индекса $k \in \mathbb{N}$ существует вектор $b_{k} \in E$ такой, что

$$
\begin{aligned}
(f \boxplus w)\left(y_{0}-a_{k}+b_{k}\right) & =f\left(y_{0}-a_{k}\right)+w\left(b_{k}\right), \\
\left(f \boxminus w_{[\mu]}\right)\left(y_{0}-a_{k}-\mu b_{k}\right) & =f\left(y_{0}-a_{k}\right)-w_{[\mu]}\left(\mu b_{k}\right) .
\end{aligned}
$$

В силу леммы 3.2 для любого $k \in \mathbb{N}$ справедливы неравенства

$$
\begin{gathered}
(f \boxplus w)\left(y_{0}-a_{k}+b_{k}\right)-\left(f \boxminus w_{[\mu]}\right)\left(y_{0}-a_{1}-\mu b_{1}\right) \\
\leqslant\left(w \boxplus w_{[\mu]}\right)\left(b_{k}-a_{k}+a_{1}+\mu b_{1}\right), \\
(f \boxplus g)\left(y_{0}\right)-\left(f \boxminus w_{[\mu]}\right)\left(y_{0}-a_{k}-\mu b_{k}\right) \leqslant\left(g \boxplus w_{[\mu]}\right)\left(\mu b_{k}+a_{k}\right) .
\end{gathered}
$$

Из соотношений $(3.10),(3.15),(3.17)$ следует, что

$$
w_{[\mu]}\left(\mu b_{k}\right)+g\left(a_{k}\right) \leqslant\left(w_{[\mu]} \boxplus g\right)\left(\mu b_{k}+a_{k}\right)+\frac{1}{k} \quad \forall k \in \mathbb{N} .
$$


Соотношения (3.14)-(3.16) дают

$f\left(y_{0}-a_{k}\right)-f\left(y_{0}-a_{1}\right)+w\left(b_{k}\right)+w_{[\mu]}\left(\mu b_{1}\right) \leqslant w_{[1+\mu]}\left(b_{k}-a_{k}+a_{1}+\mu b_{1}\right) \quad \forall k \in \mathbb{N}$.

Из ограниченности функции $f$ на любом ограниченном множестве (лемма 3.9 ), ограниченности последовательности $\left\{a_{k}\right\}$ и соотношения (3.19) следует, что последовательность $\left\{w\left(b_{k}\right)-w_{[1+\mu]}\left(b_{k}-a_{k}+a_{1}+\mu b_{1}\right)\right\}$ ограничена сверху. Поэтому согласно лемме 3.5 последовательность $\left\{b_{k}\right\}$ ограничена.

В силу леммы 3.3 для любого индекса $k \in \mathbb{N}$ существует вектор $a_{k}^{\prime} \in E$ такой, что

$$
\left(w_{[\mu]} \boxplus g\right)\left(\mu b_{k}+a_{k}\right)=w_{[\mu]}\left(\mu b_{k}+a_{k}-a_{k}^{\prime}\right)+g\left(a_{k}^{\prime}\right) .
$$

Отсюда и из соотношения (3.18) в силу леммы 3.7 имеем

$$
\lim _{k \rightarrow \infty}\left\|a_{k}-a_{k}^{\prime}\right\|=0
$$

Для любого индекса $k \in \mathbb{N}$ определим вектор

$$
b_{k}^{\prime}=b_{k}+\frac{a_{k}-a_{k}^{\prime}}{\mu} .
$$

В силу равенства (3.20) и леммы 3.6 имеем

$$
w_{[\lambda]}\left(\lambda b_{k}^{\prime}\right)=g\left(a_{k}^{\prime}\right)+\left(w_{[\lambda]} \boxminus g\right)\left(\lambda b_{k}^{\prime}-a_{k}^{\prime}\right) \quad \forall k \in \mathbb{N} .
$$

Из соотношения (3.14) следует, что

$$
f\left(y_{0}-a_{k}\right)+w\left(b_{n}+a_{k}-a_{n}\right) \geqslant f\left(y_{0}-a_{n}\right)+w\left(b_{n}\right) \quad \forall k, n \in \mathbb{N} .
$$

Следовательно, согласно неравенствам (3.10) для любых $k, n \in \mathbb{N}$ имеем

$f\left(y_{0}-a_{n}\right)+w\left(b_{n}\right)-w\left(b_{n}+a_{k}-a_{n}\right)+g\left(a_{k}\right) \leqslant(f \boxplus g)\left(y_{0}\right)+\frac{1}{k} \leqslant f\left(y_{0}-a_{n}^{\prime}\right)+g\left(a_{n}^{\prime}\right)+\frac{1}{k}$, поэтому

$$
w\left(b_{n}\right)+g\left(a_{k}\right) \leqslant w\left(b_{n}+a_{k}-a_{n}\right)+g\left(a_{n}^{\prime}\right)+\delta_{n k} \quad \forall k, n \in \mathbb{N},
$$

где $\delta_{n k}=\frac{1}{k}+f\left(y_{0}-a_{n}^{\prime}\right)-f\left(y_{0}-a_{n}\right)$. Согласно лемме 3.9 функция $f$ на любом ограниченном множестве удовлетворяет условию Липшица. Отсюда и из соотношения (3.21) следует, что

$$
\lim _{\substack{n \rightarrow \infty \\ k \rightarrow \infty}} \delta_{n k}=0 .
$$

По определению эпиразности имеем

$$
w_{[\lambda]}\left(\lambda b_{n}^{\prime}+a_{k}-a_{n}^{\prime}\right) \leqslant g\left(a_{k}\right)+\left(w_{[\lambda]} \boxminus g\right)\left(\lambda b_{n}^{\prime}-a_{n}^{\prime}\right) \quad \forall k, n \in \mathbb{N} .
$$

Отсюда и из соотношения (3.23) получаем

$$
w_{[\lambda]}\left(\lambda b_{n}^{\prime}+a_{k}-a_{n}^{\prime}\right)-w_{[\lambda]}\left(\lambda b_{n}^{\prime}\right) \leqslant g\left(a_{k}\right)-g\left(a_{n}^{\prime}\right) \quad \forall k, n \in \mathbb{N} .
$$


Следовательно, согласно неравенствам (3.24) имеем

$$
w_{[\lambda]}\left(\lambda b_{n}^{\prime}+a_{k}-a_{n}^{\prime}\right)-w_{[\lambda]}\left(\lambda b_{n}^{\prime}\right) \leqslant w\left(b_{n}+a_{k}-a_{n}\right)-w\left(b_{n}\right)+\delta_{n k} \quad \forall k, n \in \mathbb{N},
$$

поэтому

$$
\lambda w\left(b_{n}+\frac{a_{k}-a_{n}}{\lambda}\right)+(1-\lambda) w\left(b_{n}\right) \leqslant w\left(b_{n}+a_{k}-a_{n}\right)+\delta_{n k}^{\prime} \quad \forall k, n \in \mathbb{N},
$$

где

$$
\delta_{n k}^{\prime}=\delta_{n k}+w_{[\lambda]}\left(\lambda b_{n}+a_{k}-a_{n}\right)-w_{[\lambda]}\left(\lambda b_{n}^{\prime}+a_{k}-a_{n}^{\prime}\right)+w_{[\lambda]}\left(\lambda b_{n}^{\prime}\right)-w_{[\lambda]}\left(\lambda b_{n}\right) .
$$

Поскольку согласно замечанию 2.2 функция $w$ на любом ограниченном множестве удовлетворяет условию Липшица, из ограниченности последовательностей $\left\{a_{n}\right\},\left\{b_{n}\right\}$ и соотношений (3.21), (3.22), (3.25) получаем равенство

$$
\lim _{\substack{n \rightarrow \infty \\ k \rightarrow \infty}} \delta_{n k}^{\prime}=0 .
$$

Поэтому в силу соотношения (3.26), ограниченности последовательностей $\left\{a_{k}\right\}$, $\left\{b_{k}\right\}$ и равномерной выпуклости функции $w$ на любом ограниченном множестве последовательность $\left\{a_{k}\right\}$ фундаментальна, а значит, сходится. Лемма доказана.

Лemma 3.11. Пусть $w \in \mathfrak{C} \mathfrak{B}(E), \mu>0, f \in \operatorname{WConv}(w) \cap \operatorname{WConc}\left(w_{[\mu]}\right)$, $\lambda \in(0,1), g \in \operatorname{SConv}\left(w_{[\lambda]}\right), y_{0} \in E$. Тогда минимум $\min _{a \in E}(f(y-a)+g(a))$ достигается в единственной точке.

ДоказАтельство. Поскольку $f \boxplus w \boxminus w=f$, то $\operatorname{dom}(f \boxplus w) \neq \varnothing$. Отсюда и из равенств $f \boxplus w=f \boxplus w_{[\lambda]} \boxplus w_{[1-\lambda]}, \operatorname{dom} w_{[1-\lambda]}=E$ имеем $\left(f \boxplus w_{[\lambda]}\right)(y)>-\infty$ для любого $y \in E$. Следовательно, учитывая включение $g \in \operatorname{SConv}\left(w_{[\lambda]}\right)$, получаем, что $(f \boxplus g)\left(y_{0}\right)>-\infty$. Поэтому в силу определения эписуммы для любого $k \in \mathbb{N}$ существует вектор $a_{k} \in E$ такой, что

$$
f\left(y_{0}-a_{k}\right)+g\left(a_{k}\right) \leqslant(f \boxplus g)\left(y_{0}\right)+\frac{1}{k} .
$$

В силу леммы 3.10 последовательность $\left\{a_{k}\right\}$ сходится. Введем обозначение: $a_{*}=\lim _{k \rightarrow \infty} a_{k}$. Переходя к пределу в неравенстве $(3.27)$ и используя непрерывность функции $f$ и полунепрерывность снизу функции $g$, получаем неравенство

$$
f\left(y_{0}-a_{*}\right)+g\left(a_{*}\right) \leqslant(f \boxplus g)\left(y_{0}\right) .
$$

Если вектор $a \in E$ удовлетворяет неравенству $f\left(y_{0}-a\right)+g(a) \leqslant(f \boxplus g)\left(y_{0}\right)$, то в силу сходимости последовательности

$$
a_{k}^{\prime}= \begin{cases}a_{*}, & k \text { четно } \\ a, & k \text { нечетно }\end{cases}
$$

получаем равенство $a=a_{*}$. Поэтому минимум $\min _{a \in E}(f(y-a)+g(a))$ достигается в единственной точке $a=a_{*}$. Лемма доказана. 
Лемма 3.12. Пусть $w \in \mathfrak{C} \mathfrak{B}(E), \mu>0, f \in \operatorname{WConv}(w) \cap \operatorname{WConc}\left(w_{[\mu]}\right)$, $\lambda \in(0,1), g \in \operatorname{SConv}\left(w_{[\lambda]}\right)$. Tогда $f \boxplus g \in \operatorname{WConv}\left(w_{[1-\lambda]}\right)$.

ДокАЗАтЕльство. Обозначим $h=f \boxplus g, \psi=w \boxminus g$. Зафиксируем произвольный вектор $y_{0} \in E$. В силу леммы 3.11 существуют векторы $a_{0}, x_{0} \in E$ такие, что $x_{0}+a_{0}=y_{0}$ и

$$
(f \boxplus g)\left(y_{0}\right)=f\left(x_{0}\right)+g\left(a_{0}\right) .
$$

Согласно лемме 3.9 существует вектор $b_{0} \in E$, удовлетворяющий равенствам

$$
\begin{aligned}
(f \boxplus w)\left(x_{0}+b_{0}\right) & =f\left(x_{0}\right)+w\left(b_{0}\right), \\
\left(f \boxminus w_{[\mu]}\right)\left(x_{0}-\mu b_{0}\right) & =f\left(x_{0}\right)-w_{[\mu]}\left(\mu b_{0}\right) .
\end{aligned}
$$

В силу леммы 2.5 справедливо включение $\operatorname{SConv}\left(w_{[\lambda]}\right) \subset \operatorname{SConv}(w)$. Поэтому $g \in \operatorname{SConv}(w)$, а значит, $\psi \boxplus g=w$. Из соотношений (3.28) и (3.30) в силу леммы 3.2 и определения эписуммы получаем равенство

$$
g\left(a_{0}\right)+w_{[\mu]}\left(\mu b_{0}\right)=\left(g \boxplus w_{[\mu]}\right)\left(a_{0}+\mu b_{0}\right) .
$$

Следовательно, согласно лемме 3.6 имеем $(w \boxminus g)\left(b_{0}-a_{0}\right)=w\left(b_{0}\right)-g\left(a_{0}\right)$, т. е.

$$
\psi\left(b_{0}-a_{0}\right)=w\left(b_{0}\right)-g\left(a_{0}\right) .
$$

Отсюда и из равенств (3.29) и $f \boxplus w=f \boxplus g \boxplus \psi=h \boxplus \psi$ следует, что

$$
(h \boxplus \psi)\left(x_{0}+b_{0}\right)=f\left(x_{0}\right)+g\left(a_{0}\right)+\psi\left(b_{0}-a_{0}\right) .
$$

Поэтому согласно равенствам $x_{0}+a_{0}=y_{0}$ и (3.28) имеем

$$
(h \boxplus \psi)\left(y_{0}+b_{0}-a_{0}\right)=h\left(y_{0}\right)+\psi\left(b_{0}-a_{0}\right) .
$$

Согласно замечанию 2.4 получаем включение

$$
h \in \operatorname{WConv}(\psi) .
$$

Поскольку $g \in \operatorname{SConv}\left(w_{[\lambda]}\right)$, функция $\eta=w_{[\lambda]} \boxminus g$ удовлетворяет равенству $w_{[\lambda]}=\eta \boxplus g$. Следовательно, $\psi=w \boxminus g=w_{[1-\lambda]} \boxplus \eta \boxplus g \boxminus g$. Так как $w_{[\lambda]}=$ $\eta \boxplus g$, то согласно лемме 3.4 функция $g$ коэрцитивна. Из леммы 3.3 следует, что $w_{[1-\lambda]} \boxplus \eta \in \mathfrak{C}(E)$. В силу леммы 2.7 справедливо равенство $w_{[1-\lambda]} \boxplus \eta \boxplus$ $g \boxminus g=w_{[1-\lambda]} \boxplus \eta$. Поэтому $\psi=w_{[1-\lambda]} \boxplus \eta$, а значит, $w_{[1-\lambda]} \in \operatorname{SConv}(\psi)$. Отсюда и из леммы 2.3 следует, что $\operatorname{WConv}(\psi) \subset \operatorname{WConv}\left(w_{[1-\lambda]}\right)$. Применяя включение (3.31), получаем включение $h \in \operatorname{WConv}\left(w_{[1-\lambda]}\right)$. Лемма доказана.

Теорема 3.1 (исчисление параметров выпуклости функций). Пусть заданы положительные числа $R, T, t$ и функции

$$
w \in \mathfrak{C} \mathfrak{B}(E), \quad f \in \operatorname{WConv}\left(w_{[R]}\right) \cap \operatorname{WConc}\left(w_{[T]}\right), \quad h \in \operatorname{SConv}\left(w_{[t]}\right) .
$$

Тогда:

(i) если $t<R$, mo $f \boxplus h \in \operatorname{WConv}\left(w_{[R-t]}\right) \cap \operatorname{WConc}\left(w_{[T]}\right)$;

(ii) если $t<T$, mo $f \boxminus h \in \mathrm{WConv}\left(w_{[R]}\right) \cap \operatorname{WConc}\left(w_{[T-t]}\right)$. 
Доказательство. Докажем утверждение (i). Пусть $t<R$. Включение $f \boxplus h \in \mathrm{WConv}\left(w_{[R-t]}\right)$ следует из леммы 3.12. Включение $f \boxplus h \in \operatorname{WConc}\left(w_{[T]}\right)$ следует из леммы 2.4,(ii).

Докажем утверждение (ii). Пусть $t<T$. Рассмотрим функцию $\widetilde{f}$, определяемую равенством $\widetilde{f}(x)=-f(-x)$ при любом $x \in E$. Тогда имеем включение $\widetilde{f} \in \operatorname{WConv}\left(w_{[T]}\right) \cap \operatorname{WConc}\left(w_{[R]}\right)$. В силу утверждения (i) справедливо включение $\tilde{f} \boxplus h \in \operatorname{WConv}\left(w_{[T-t]}\right) \cap \operatorname{WConc}\left(w_{[R]}\right)$. Отсюда и из равенства $-(\tilde{f} \boxplus h)(-x)=(f \boxminus h)(x)$ следует утверждение (ii). Теорема доказана.

ЗАмЕЧАНИЕ 3.1. Оценки параметров выпуклости эписуммы и эпиразности функций, приведенные в теореме 3.1, неулучшаемы. Это следует из свойства (ix) эпиопераций.

ЗАмечАниЕ 3.2. Согласно теореме 3.1 параметр слабой выпуклости функции $f \boxplus h$ равен разности параметра слабой выпуклости функции $f$ и параметра сильной выпуклости функции $h$. Однако при этом существенно, что функция $f$ слабо вогнута с некоторым параметром. Следующий пример показывает, что из условий $w \in \mathfrak{C} \mathfrak{B}(E), f \in \operatorname{WConv}\left(w_{[R]}\right), h \in \operatorname{SConv}\left(w_{[t]}\right), 0<t<R$, не следует включение $f \boxplus h \in \mathrm{WConv}\left(w_{[R-t]}\right)$.

ПримеР 3.1. Пусть $E=\mathbb{R}^{2}$,

$$
\begin{gathered}
w\left(x_{1}, x_{2}\right)=\left(x_{1}^{2}+x_{2}^{2}\right)^{2}, \quad f\left(x_{1}, x_{2}\right)=-\left(x_{1}^{2}+\left(\left|x_{2}\right|-1\right)^{2}\right)^{2}, \\
h=w_{[1 / 2]}, \quad t=\frac{1}{2}, \quad R=1 .
\end{gathered}
$$

Тогда $w \in \mathfrak{C} \mathfrak{B}(E), h \in \operatorname{SConv}\left(w_{[t]}\right)$. Поскольку

$$
(f \boxplus w)\left(x_{1}, x_{2}\right)=\left\{\begin{array}{ll}
0, & \left(x_{1}, x_{2}\right) \in\{(0,1),(0,-1)\}, \\
-\infty, & \left(x_{1}, x_{2}\right) \notin\{(0,1),(0,-1)\},
\end{array} \quad f \boxplus w \boxminus w=f,\right.
$$

то $f \in \operatorname{WConv}\left(w_{[R]}\right)$, а поскольку $f \boxplus h \boxplus w_{[R-t]} \boxminus w_{[R-t]}=f \boxplus w \boxminus w_{[1 / 2]}$, то

$\left(f \boxplus h \boxplus w_{[R-t]} \boxminus w_{[R-t]}\right)(0,0)=\max \left\{-w_{[1 / 2]}(0,-1),-w_{[1 / 2]}(0,1)\right\}=-8$.

С другой стороны,

$$
(f \boxplus h)(0,0)=\inf _{\substack{u_{1} \in \mathbb{R} \\ u_{2} \in \mathbb{R}}}\left(-\left(u_{1}^{2}+\left(\left|u_{2}\right|-1\right)^{2}\right)^{2}+8\left(u_{1}^{2}+u_{2}^{2}\right)^{2}\right)=-\frac{8}{7}>-8 .
$$

Поэтому $f \boxplus h \boxplus w_{[R-t]} \boxminus w_{[R-t]} \neq f \boxplus h$, а значит, $f \boxplus h \notin \operatorname{WConv}\left(w_{[R-t]}\right)$.

\section{§4. Доказательство теоремы о седловой точке}

Пусть выполнены предположения теоремы 1.1. Выберем натуральное число $n$ такое, что $\frac{t}{n}<R-r$. Для любого $k \in\{1, \ldots, n\}$ определим функцию $h_{k}=h_{[k / n]}$. Поскольку $h \in \operatorname{SConv}\left(w_{[t]}\right)$, то согласно лемме 2.6,(i) имеем включение $h_{k} \in \operatorname{SConv}\left(w_{[k t / n]}\right)$. Положим

$$
h_{0}(x)= \begin{cases}0, & x=0 \\ +\infty, & x \neq 0\end{cases}
$$


Покажем, что для любого $k \in\{0,1, \ldots, n\}$ справедливо равенство

$$
f \boxminus h_{k} \boxplus g=f \boxplus g \boxminus h_{k} .
$$

При $k=0$ равенство (4.1) справедливо. Пусть равенство (4.1) справедливо при $k=s \in\{0,1, \ldots, n-1\}$. Покажем, что равенство (4.1) справедливо при $k=s+1$. В силу теоремы 3.1 имеем $f \boxminus h_{s+1} \boxplus g \in \operatorname{WConv}\left(w_{[R-r]}\right)$. Отсюда и из включений $h_{1} \in \operatorname{SConv}\left(w_{[t / n]}\right) \subset \operatorname{SConv}\left(w_{[R-r]}\right)$ в силу леммы 2.3 получаем включение $f \boxminus h_{s+1} \boxplus g \in \mathrm{WConv}\left(h_{1}\right)$. Поэтому справедливо равенство

$$
f \boxminus h_{s+1} \boxplus g \boxplus h_{1} \boxminus h_{1}=f \boxminus h_{s+1} \boxplus g,
$$

а следовательно, равенство

$$
f \boxminus h_{s+1} \boxplus h_{1} \boxplus g \boxminus h_{1}=f \boxminus h_{s+1} \boxplus g .
$$

В силу теоремы 3.1 справедливо включение $f \boxminus h_{s} \in \operatorname{WConc}\left(w_{\left[T-\frac{s}{n} t\right]}\right)$. Отсюда и из включений $h_{1} \in \operatorname{SConv}\left(w_{[t / n]}\right) \subset \operatorname{SConv}\left(w_{\left[T-\frac{s}{n} t\right]}\right)$ согласно лемме 2.3 следует, что $f \boxminus h_{s} \in \operatorname{WConc}\left(h_{1}\right)$, а значит, $f \boxminus h_{s}=f \boxminus h_{s} \boxminus h_{1} \boxplus h_{1}=f \boxminus h_{s+1} \boxplus h_{1}$. Отсюда и из равенства (4.2) получаем

$$
f \boxminus h_{s} \boxplus g \boxminus h_{1}=f \boxminus h_{s+1} \boxplus g .
$$

Поэтому, используя равенство (4.1) при $k=s$, имеем

$$
f \boxplus g \boxminus h_{s} \boxminus h_{1}=f \boxminus h_{s+1} \boxplus g,
$$

что доказывает равенство (4.1) при $k=s+1$. По индукции получаем, что равенство (4.1) справедливо для любого $k \in\{0,1, \ldots, n\}$. В частности, имеем $f \boxminus h_{n} \boxplus g=f \boxplus g \boxminus h_{n}$, т. е.

$$
f \boxminus h \boxplus g=f \boxplus g \boxminus h .
$$

Зафиксируем произвольный вектор $x \in E$. Поскольку согласно теореме 3.1 имеем $f \boxminus h \in \operatorname{WConv}\left(w_{[R]}\right) \cap \operatorname{WConv}\left(w_{[T-t]}\right)$, в силу леммы 3.11 существует вектор $u_{*} \in E$ такой, что

$$
(f \boxminus h \boxplus g)(x)=(f \boxminus h)\left(x-u_{*}\right)+g\left(u_{*}\right) .
$$

Аналогично, существует вектор $v_{*}$ такой, что

$$
(f \boxplus g \boxminus h)(x)=(f \boxplus g)\left(x+v_{*}\right)-h\left(v_{*}\right) .
$$

Из равенств (4.3)-(4.5) следует, что

$$
(f \boxminus h)\left(x-u_{*}\right)+g\left(u_{*}\right)=(f \boxplus g)\left(x+v_{*}\right)-h\left(v_{*}\right) .
$$

Поэтому по определению эпиопераций получаем

$$
f\left(x-u_{*}+v\right)+g\left(u_{*}\right)-h(v) \leqslant f\left(x-u+v_{*}\right)+g(u)-h\left(v_{*}\right) \quad \forall u, v \in E .
$$

Следовательно, пара $\left(u_{*}, v_{*}\right)$ составляет седловую точку функции (1.1). В силу леммы 3.11 седловая точка этой функции единственна. Теорема 1.1 доказана. 


\section{§ 5. Непрерывная зависимость седловой точки от параметров}

Будем говорить, что последовательность функций $f_{k}: E \rightarrow \mathbb{R} \cup\{+\infty\}$ сходится $\kappa$ функиии $f: E \rightarrow \mathbb{R} \cup\{+\infty\}$ в смысле $\mathfrak{C} \mathfrak{B}, f_{k} \underset{\mathfrak{C} \mathfrak{B}}{\longrightarrow} f$, если для любой ограниченной последовательности векторов $x_{k} \in \operatorname{dom} f_{k}$ найдется последовательность векторов $z_{k} \in \operatorname{dom} f$ такая, что

$$
\lim _{k \rightarrow \infty}\left\|x_{k}-z_{k}\right\|=0, \quad \lim _{k \rightarrow \infty}\left|f_{k}\left(x_{k}\right)-f\left(z_{k}\right)\right|=0,
$$

и, кроме того, для любой ограниченной последовательности векторов $z_{k} \in$ $\operatorname{dom} f$ найдется последовательность векторов $x_{k} \in \operatorname{dom} f_{k}$, для которой справедливы соотношения (5.1).

ЗАмечАниЕ 5.1. Если на любом ограниченном множестве $B \subset E$ последовательность функций $f_{k}: E \rightarrow \mathbb{R}$ равномерно сходится к функции $f: E \rightarrow \mathbb{R}$, то, как легко видеть, $f_{k} \underset{\mathfrak{C B}}{\longrightarrow} f$. При этом на всем пространстве $E$ последовательность $\left\{f_{k}\right\}$ может сходиться неравномерно. Например, пусть $E=\mathbb{R}$, $f_{k}(x)=c_{k} x^{2}, f(x)=c x^{2}$, где числовая последовательность $\left\{c_{k}\right\}$ сходится к числу с. Тогда $f_{k} \underset{\mathfrak{C B}}{\longrightarrow} f$, но на всем пространстве $E=\mathbb{R}$ последовательность $\left\{f_{k}\right\}$ сходится неравномерно.

ЗАмЕчАниЕ 5.2. Если надграфики функций $f_{k}$ сходятся в смысле метрики Хаусдорфа к надграфику функции $f$, то, как легко видеть, $f_{k} \underset{\mathfrak{c} \mathfrak{B}}{\longrightarrow} f$. При этом в фиксированной точке $x \in E$ сходимость $f_{k}(x) \rightarrow f(x)$ может не иметь места. Пусть, например,

$$
E=\mathbb{R}, \quad f_{k}(x)=\left\{\begin{array}{ll}
0, & x \in\left[\frac{1}{k}, 1\right], \\
+\infty, & x \notin\left[\frac{1}{k}, 1\right],
\end{array} \quad f(x)= \begin{cases}0, & x \in[0,1], \\
+\infty, & x \notin[0,1] .\end{cases}\right.
$$

Тогда $f_{k} \underset{\mathfrak{C B}}{\longrightarrow} f$, но $f_{k}(0) \nrightarrow f(0)$.

Лемма 5.1. Пусть $w \in \mathfrak{C} \mathfrak{B}(E)$, и пусть последовательность функиий $g_{k} \in \operatorname{SConv}(w)$ сходится в смвиле $\mathfrak{C} \mathfrak{B} \kappa$ функции $g_{0} \in \operatorname{SConv}(w)$. Тогда существует ограниченная последовательность векторов $\left\{z_{k}\right\} \subset E$ такая, что последовательность $\left\{\left(w \boxminus g_{k}\right)\left(z_{k}\right)\right\}$ ограничена сверху.

ДоказАтельство. Для любого индекса $k \in \mathbb{N} \cup\{0\}$ обозначим $\varphi_{k}=w \boxminus g_{k}$. Поскольку $g_{k} \in \operatorname{SConv}(w)$, то $g_{k} \boxplus \varphi_{k}=w$ при всех $k \in \mathbb{N} \cup\{0\}$. Так как функция $w$ коэрцитивна, то согласно лемме 3.4 функции $g_{k}, \varphi_{k}$ коэрцитивны при всех $k \in \mathbb{N} \cup\{0\}$. Поэтому в силу выпуклости и полунепрерывности снизу этих функций существуют векторы $x_{k}, z_{k} \in E$ такие, что

$$
g_{k}\left(x_{k}\right)=\min _{x \in E} g_{k}(x), \quad \varphi_{k}\left(z_{k}\right)=\min _{x \in E} \varphi_{k}(x) \quad \forall k \in \mathbb{N} \cup\{0\} .
$$

Отсюда и из равенств $g_{k} \boxplus \varphi_{k}=w$ следует, что

$$
g_{k}\left(x_{k}\right)+\varphi_{k}\left(z_{k}\right)=w\left(x_{k}+z_{k}\right)=\min _{x \in E} w(x) \quad \forall k \in \mathbb{N} \cup\{0\} .
$$


Поэтому с учетом строгой выпуклости функции $w$ получаем равенства

$$
x_{k}+z_{k}=x_{0}+z_{0} \quad \forall k \in \mathbb{N} \text {. }
$$

Поскольку $g_{k} \underset{\mathfrak{C B}}{\longrightarrow} g_{0}$, существует последовательность $\left\{a_{k}\right\} \subset E$ такая, что

$$
\lim _{k \rightarrow \infty}\left\|a_{k}-x_{0}\right\|=0, \quad \lim _{k \rightarrow \infty}\left|g_{k}\left(a_{k}\right)-g_{0}\left(x_{0}\right)\right|=0 .
$$

Покажем, что $\lim _{k \rightarrow \infty}\left\|x_{k}-a_{k}\right\|=0$. Предположим противное. Тогда, выделяя подпоследовательность, без потери общности будем считать, что справедливы неравенства $\left\|x_{k}-a_{k}\right\| \geqslant \varepsilon_{0}>0$ для любого $k \in \mathbb{N}$. При всех $k \in \mathbb{N}$ определим

$$
u_{k}=\left(1-\frac{\varepsilon_{0}}{\left\|x_{k}-a_{k}\right\|}\right) a_{k}+\frac{\varepsilon_{0}}{\left\|x_{k}-a_{k}\right\|} x_{k} .
$$

Тогда $\left\|u_{k}-a_{k}\right\|=\varepsilon_{0}$ и в силу выпуклости функции $g_{k}$ имеем

$$
g_{k}\left(u_{k}\right) \leqslant \max \left\{g_{k}\left(a_{k}\right), g_{k}\left(x_{k}\right)\right\} \quad \forall k \in \mathbb{N} .
$$

Отсюда и из соотношений $g_{k}\left(x_{k}\right)=\min _{x \in E} g_{k}(x) \leqslant g\left(a_{k}\right)$ следует, что $g_{k}\left(u_{k}\right) \leqslant$ $g_{k}\left(a_{k}\right)$. Поскольку последовательность $\left\{u_{k}\right\}$ ограничена и $g_{k} \underset{\mathfrak{C B}}{\longrightarrow} g_{0}$, существует последовательность $\left\{v_{k}\right\} \subset E$ такая, что

$$
\lim _{k \rightarrow \infty}\left\|v_{k}-u_{k}\right\|=0, \quad \lim _{k \rightarrow \infty}\left|g_{0}\left(v_{k}\right)-g_{k}\left(u_{k}\right)\right|=0 .
$$

Следовательно,

$$
\lim _{k \rightarrow \infty}\left\|v_{k}-x_{0}\right\|=\varepsilon_{0}, \quad \limsup _{k \rightarrow \infty} g_{0}\left(v_{k}\right) \leqslant g_{0}\left(x_{0}\right) .
$$

По определению эписуммы $\left(g_{0} \boxplus \varphi_{0}\right)\left(v_{k}+z_{0}\right) \leqslant g_{0}\left(v_{k}\right)+\varphi_{0}\left(z_{0}\right)$. Следовательно, имеем $\lim \sup _{k \rightarrow \infty} w\left(v_{k}+z_{0}\right) \leqslant g_{0}\left(x_{0}\right)+\varphi_{0}\left(z_{0}\right)$. Используя равенства (5.2) при $k=0$, получаем $\lim \sup _{k \rightarrow \infty} w\left(v_{k}+z_{0}\right) \leqslant w\left(x_{0}+z_{0}\right)=\min _{x \in E} w(x)$, что вместе с соотношением $\lim _{k \rightarrow \infty}\left\|v_{k}-x_{0}\right\|=\varepsilon_{0}$ противоречит равномерной выпуклости функции $w$ на любом ограниченном множестве. Полученное противоречие доказывает соотношение $\lim _{k \rightarrow \infty}\left\|x_{k}-a_{k}\right\|=0$. Отсюда и из соотношения $\lim _{k \rightarrow \infty}\left\|a_{k}-x_{0}\right\|=0$ следует сходимость последовательности $\left\{x_{k}\right\}$.

Поскольку $g_{k} \underset{\mathfrak{C B}}{\longrightarrow} g_{0}$ и функция $g_{0}$ ограничена снизу, последовательность $g_{k}\left(x_{k}\right)$ ограничена снизу. Отсюда и из равенств (5.2) следует ограниченность сверху последовательности $\left\{\left(w \boxminus g_{k}\right)\left(z_{k}\right)\right\}=\left\{\varphi_{k}\left(z_{k}\right)\right\}$. Из равенств (5.3) и ограниченности последовательности $\left\{x_{k}\right\}$ следует ограниченность последовательности $\left\{z_{k}\right\}$. Лемма доказана.

Лемма 5.2. Пусть $w \in \mathfrak{C} \mathfrak{B}(E), \mu>0$, последовательность функиий $f_{k} \in$ $\operatorname{WConv}(w) \cap \operatorname{WConc}\left(w_{[\mu]}\right)$ сходится в смысле $\mathfrak{C} \mathfrak{B} \kappa$ функиии $f \in \operatorname{WConv}(w) \cap$ $\operatorname{WConc}\left(w_{[\mu]}\right)$. Тогда существует ограниченная последовательность векторов $\left\{b_{k}\right\} \subset E$ такая, что последовательность $\left\{\left(f_{k} \boxplus w\right)\left(b_{k}\right)\right\}$ ограничена снизу. 
ДокАЗАТЕльство. В силу леммы 3.9 для любого индекса $k \in \mathbb{N}$ существует вектор $b_{k} \in E$ такой, что

$$
\left(f_{k} \boxplus w\right)\left(b_{k}\right)=f_{k}(0)+w\left(b_{k}\right) .
$$

Предположим, что последовательность $\left\{b_{k}\right\}$ неограничена. Выделяя подпоследовательность, без потери общности будем считать, что $\lim _{k \rightarrow \infty}\left\|b_{k}\right\|=+\infty$ и $\left\|b_{k}\right\|>1$ для любого $k \in \mathbb{N}$.

Для любого $k \in \mathbb{N}$ определим вектор $u_{k}=\frac{b_{k}}{\left\|b_{k}\right\|}$. По определению эписуммы для любого $k \in \mathbb{N}$ имеем $\left(f_{k} \boxplus w\right)\left(b_{k}\right) \leqslant f_{k}\left(u_{k}\right)+w\left(b_{k}-u_{k}\right)$. Отсюда и из равенств (5.4) получаем

$$
f_{k}(0)+w\left(b_{k}\right) \leqslant f_{k}\left(u_{k}\right)+w\left(b_{k}-u_{k}\right) \quad \forall k \in \mathbb{N} .
$$

Поскольку последовательность $\left\{u_{k}\right\}$ ограничена, $f_{k} \underset{\mathfrak{C} \mathfrak{B}}{\longrightarrow} f$ и согласно лемме 3.9 функция $f$ ограничена на любом ограниченном множестве, последовательности $\left\{f_{k}\left(u_{k}\right)\right\}$ и $\left\{f_{k}(0)\right\}$ ограничены. Отсюда и из соотношения (5.5) следует, что

$$
\sup _{k \in \mathbb{N}}\left(w\left(b_{k}\right)-w\left(b_{k}-u_{k}\right)\right)<+\infty .
$$

Используя соотношение $\lim _{k \rightarrow \infty}\left\|b_{k}\right\|=+\infty$ и коэрцитивность функции $w$, получаем

$$
\lim _{k \rightarrow \infty} \frac{w\left(b_{k}\right)-w(0)}{\left\|b_{k}\right\|}=+\infty .
$$

В силу выпуклости функции $w$ имеем

$$
w\left(b_{k}\left(1-\frac{1}{\left\|b_{k}\right\|}\right)\right) \leqslant\left(1-\frac{1}{\left\|b_{k}\right\|}\right) w\left(b_{k}\right)+\frac{1}{\left\|b_{k}\right\|} w(0) \quad \forall k \in \mathbb{N},
$$

то есть

$$
\frac{w\left(b_{k}\right)-w(0)}{\left\|b_{k}\right\|} \leqslant w\left(b_{k}\right)-w\left(b_{k}-u_{k}\right) \quad \forall k \in \mathbb{N} .
$$

Отсюда и из соотношения (5.7) получаем равенство

$$
\lim _{k \rightarrow \infty}\left(w\left(b_{k}\right)-w\left(b_{k}-u_{k}\right)\right)=+\infty
$$

которое противоречит неравенству (5.6). Полученное противоречие доказывает ограниченность последовательности $\left\{b_{k}\right\}$. Из ограниченности последовательности $\left\{b_{k}\right\}$, равенств (5.4) и соотношения $f_{k} \underset{\mathfrak{C B}}{\longrightarrow} f$ следует, что последовательность $\left\{\left(f_{k} \boxplus w\right)\left(b_{k}\right)\right\}$ ограничена. Лемма доказана.

Лемма 5.3. Пусть $w \in \mathfrak{C B}(E), \mu>0$, последовательность функиий $f_{k} \in$ $\operatorname{WConv}(w) \cap \operatorname{WConc}\left(w_{[\mu]}\right)$ сходится в смысле $\mathfrak{C} \mathfrak{B} \kappa$ функиии $f \in \operatorname{WConv}(w) \cap$ $\operatorname{WConc}\left(w_{[\mu]}\right)$. Пусть $\lambda \in(0,1)$ и последовательность функиий $g_{k} \in \operatorname{SConv}\left(w_{[\lambda]}\right)$ сходится в смысле $\mathfrak{C} \mathfrak{B} \kappa$ функиии $g \in \operatorname{SConv}\left(w_{[\lambda]}\right)$. Пусть заданы последовательности $\left\{a_{k}\right\} \subset E u\left\{y_{k}\right\} \subset E$ такие, что

$$
f_{k}\left(y_{k}-a_{k}\right)+g_{k}\left(a_{k}\right) \leqslant\left(f_{k} \boxplus g_{k}\right)\left(y_{k}\right)+\frac{1}{k} \quad \forall k \in \mathbb{N} .
$$


Тогда:

(i) если последовательность $\left\{y_{k}\right\}$ ограничена, то последовательность $\left\{a_{k}\right\}$ также ограничена;

(ii) если последовательность $\left\{y_{k}\right\}$ сходится, то последовательность $\left\{a_{k}\right\}$ также сходится.

Доказательство. (i) Пусть последовательность $\left\{y_{k}\right\}$ ограничена. Зафиксируем произвольный вектор $a_{0} \in \operatorname{dom} g$. Поскольку $g_{k} \underset{\mathfrak{C B}}{\longrightarrow} g$, существует последовательность векторов $a_{k}^{\prime} \in \operatorname{dom} g_{k}$ таких, что

$$
\lim _{k \rightarrow \infty}\left\|a_{k}^{\prime}-a_{0}\right\|=0, \quad \lim _{k \rightarrow \infty}\left|g_{k}\left(a_{k}^{\prime}\right)-g\left(a_{0}\right)\right|=0 .
$$

По определению эписуммы имеем

$$
\left(f_{k} \boxplus g_{k}\right)\left(y_{k}\right) \leqslant f_{k}\left(y_{k}-a_{k}^{\prime}\right)+g_{k}\left(a_{k}^{\prime}\right) \quad \forall k \in \mathbb{N} .
$$

Отсюда и из неравенств (5.8) следует, что

$$
f_{k}\left(y_{k}-a_{k}\right)+g_{k}\left(a_{k}\right) \leqslant f_{k}\left(y_{k}-a_{k}^{\prime}\right)+g_{k}\left(a_{k}^{\prime}\right)+\frac{1}{k} \quad \forall k \in \mathbb{N} .
$$

Поэтому, используя соотношения $(5.9), f_{k} \underset{\mathfrak{C} \mathfrak{B}}{\longrightarrow} f$ и ограниченность функции $f$ на любом ограниченном множестве (лемма 3.9 ), получаем ограниченность сверху последовательности $\left\{f_{k}\left(y_{k}-a_{k}\right)+g_{k}\left(a_{k}\right)\right\}$.

В силу лемм 5.1, 5.2 существуют ограниченные последовательности $\left\{z_{k}\right\}$, $\left\{b_{k}\right\} \subset E$ такие, что последовательность $\left\{\left(w_{[\lambda]} \boxminus g_{k}\right)\left(z_{k}\right)\right\}$ ограничена сверху, а последовательность $\left\{\left(f_{k} \boxplus w\right)\left(b_{k}\right)\right\}$ ограничена снизу. По определению эписуммы и эпиразности имеем

$$
\begin{gathered}
\left(w_{[\lambda]} \boxminus g_{k}\right)\left(z_{k}\right) \geqslant w_{[\lambda]}\left(z_{k}+a_{k}\right)-g_{k}\left(a_{k}\right) \quad \forall k \in \mathbb{N}, \\
\left(f_{k} \boxplus w\right)\left(b_{k}\right) \leqslant f_{k}\left(y_{k}-a_{k}\right)+w\left(b_{k}+a_{k}-y_{k}\right) \quad \forall k \in \mathbb{N} .
\end{gathered}
$$

Поэтому последовательность $\left\{w_{[\lambda]}\left(z_{k}+a_{k}\right)-g_{k}\left(a_{k}\right)\right\}$ ограничена сверху, а последовательность $\left\{f_{k}\left(y_{k}-a_{k}\right)+w\left(b_{k}+a_{k}-y_{k}\right)\right\}$ ограничена снизу. Следовательно, последовательность $\left\{w_{[\lambda]}\left(z_{k}+a_{k}\right)-w\left(b_{k}+a_{k}-y_{k}\right)-f_{k}\left(y_{k}-a_{k}\right)-\right.$ $\left.g_{k}\left(a_{k}\right)\right\}$ ограничена сверху. Отсюда, используя ограниченность сверху последовательности $\left\{f_{k}\left(y_{k}-a_{k}\right)+g_{k}\left(a_{k}\right)\right\}$, получаем, что последовательность $\left\{w_{[\lambda]}\left(z_{k}+a_{k}\right)-w\left(b_{k}+a_{k}-y_{k}\right)\right\}$ ограничена сверху. Применение леммы 3.5 и ограниченность последовательностей $\left\{y_{k}\right\},\left\{z_{k}\right\},\left\{b_{k}\right\}$ дает ограниченность последовательности $\left\{a_{k}\right\}$.

(ii) Пусть последовательность $\left\{y_{k}\right\}$ сходится к вектору $y_{0}$. В силу леммы 3.11 существует вектор $a_{0} \in E$ такой, что

$$
f\left(y_{0}-a_{0}\right)+g\left(a_{0}\right)=(f \boxplus g)\left(y_{0}\right) .
$$

Повторяя рассуждения из п. (i) для этого вектора $a_{0}$, получаем последовательность векторов $a_{k}^{\prime} \in \operatorname{dom} g_{k}$, удовлетворяющую соотношениям (5.9), (5.10). Поскольку $g_{k} \underset{\mathfrak{C B}}{\longrightarrow} g$, существует последовательность $\left\{a_{k}^{\prime \prime}\right\} \subset \operatorname{dom} g$ такая, что

$$
\lim _{k \rightarrow \infty}\left\|a_{k}^{\prime \prime}-a_{k}\right\|=0, \quad \lim _{k \rightarrow \infty}\left|g\left(a_{k}^{\prime \prime}\right)-g_{k}\left(a_{k}\right)\right|=0 .
$$


Для любого индекса $k \in \mathbb{N}$ определим число

$$
\varepsilon_{k}=f\left(y_{0}-a_{k}^{\prime \prime}\right)+g\left(a_{k}^{\prime \prime}\right)-f\left(y_{0}-a_{0}\right)-g\left(a_{0}\right) .
$$

Из соотношений $f_{k} \underset{\mathfrak{C B}}{\longrightarrow} f,\left\|a_{k}^{\prime \prime}-a_{k}\right\| \rightarrow 0,\left\|a_{k}^{\prime}-a_{0}\right\| \rightarrow 0,\left\|y_{k}-y_{0}\right\| \rightarrow 0$ и равномерной непрерывности функции $f$ на любом ограниченном множестве (лемма 3.9) следует, что

$$
\lim _{k \rightarrow \infty}\left|f\left(y_{0}-a_{k}^{\prime \prime}\right)-f_{k}\left(y_{k}-a_{k}\right)\right|=0, \quad \lim _{k \rightarrow \infty}\left|f\left(y_{0}-a_{0}\right)-f_{k}\left(y_{k}-a_{k}^{\prime}\right)\right|=0 .
$$

Отсюда и из соотношений (5.9), (5.12), (5.13) получаем неравенство

$$
\limsup _{k \rightarrow \infty} \varepsilon_{k} \leqslant \limsup _{k \rightarrow \infty}\left(f_{k}\left(y_{k}-a_{k}\right)+g_{k}\left(a_{k}\right)-f_{k}\left(y_{k}-a_{k}^{\prime}\right)-g_{k}\left(a_{k}^{\prime}\right)\right) .
$$

Поэтому согласно неравенству (5.10) имеем $\lim \sup _{k \rightarrow \infty} \varepsilon_{k}=0$. Применяя соотношения (5.11), (5.13), получаем

$$
f\left(y_{0}-a_{k}^{\prime \prime}\right)+g\left(a_{k}^{\prime \prime}\right) \leqslant(f \boxplus g)\left(y_{0}\right)+\varepsilon_{k} \quad \forall k \in \mathbb{N} .
$$

Отсюда в силу леммы 3.10 следует сходимость последовательности $\left\{a_{k}^{\prime \prime}\right\}$, а значит, и сходимость последовательности $\left\{a_{k}\right\}$. Лемма доказана.

Лемма 5.4. Пусть $w \in \mathfrak{C} \mathfrak{B}(E), \mu>0$, и пусть последовательность функций $f_{k} \in \operatorname{WConv}(w) \cap \operatorname{WConc}\left(w_{[\mu]}\right)$ сходится в смысле $\mathfrak{C} \mathfrak{B}$ к функиии $f \in \operatorname{WConv}(w) \cap \operatorname{WConc}\left(w_{[\mu]}\right)$. Пусть $\lambda \in(0,1)$ и последовательность функиий $g_{k} \in \operatorname{SConv}\left(w_{[\lambda]}\right)$ сходится в смысле $\mathfrak{C} \mathfrak{B} \kappa$ функиии $g \in \operatorname{SConv}\left(w_{[\lambda]}\right)$. Тогда последовательность $\left\{f_{k} \boxplus g_{k}\right\}$ сходится в смысле $\mathfrak{C} \mathfrak{B}$ фучници $f \boxplus g$.

ДокАЗАТЕльство. Пусть задана произвольная ограниченная последовательность $\left\{x_{k}\right\} \subset E$. Покажем, что

$$
\lim _{k \rightarrow \infty}\left|\left(f_{k} \boxplus g_{k}\right)\left(x_{k}\right)-(f \boxplus g)\left(x_{k}\right)\right|=0 .
$$

В силу леммы 3.11 для любого индекса $k \in \mathbb{N}$ существуют векторы $a_{k}, b_{k} \in E$ такие, что

$$
\begin{aligned}
(f \boxplus g)\left(x_{k}\right) & =f\left(x_{k}-a_{k}\right)+g\left(a_{k}\right), \\
\left(f_{k} \boxplus g_{k}\right)\left(x_{k}\right) & =f_{k}\left(x_{k}-b_{k}\right)+g_{k}\left(b_{k}\right) .
\end{aligned}
$$

Согласно лемме 5.3,(i) последовательности $\left\{a_{k}\right\}$ и $\left\{b_{k}\right\}$ ограничены. Поскольку $g_{k} \underset{\mathfrak{C} \mathfrak{B}}{\longrightarrow} g$, существуют последовательности векторов $a_{k}^{\prime} \in \operatorname{dom} g_{k}$ и $b_{k}^{\prime} \in \operatorname{dom} g$ такие, что

$$
\begin{aligned}
& \lim _{k \rightarrow \infty}\left\|a_{k}^{\prime}-a_{k}\right\|=0, \quad \lim _{k \rightarrow \infty}\left\|b_{k}^{\prime}-b_{k}\right\|=0, \\
& \lim _{k \rightarrow \infty}\left|g_{k}\left(a_{k}^{\prime}\right)-g\left(a_{k}\right)\right|=0, \quad \lim _{k \rightarrow \infty}\left|g\left(b_{k}^{\prime}\right)-g_{k}\left(b_{k}\right)\right|=0 \text {. }
\end{aligned}
$$

Из соотношений $f_{k} \underset{\mathfrak{C B}}{\longrightarrow} f,(5.17)$ и равномерной непрерывности функции $f$ на любом ограниченном множестве (лемма 3.9 ) следует, что

$$
\lim _{k \rightarrow \infty}\left|f_{k}\left(x_{k}-a_{k}^{\prime}\right)-f\left(x_{k}-a_{k}\right)\right|=0, \quad \lim _{k \rightarrow \infty}\left|f\left(x_{k}-b_{k}^{\prime}\right)-f_{k}\left(x_{k}-b_{k}\right)\right|=0 .
$$


Поскольку по определению эписуммы выполняются неравенства

$$
\left(f_{k} \boxplus g_{k}\right)\left(x_{k}\right) \leqslant f_{k}\left(x_{k}-a_{k}^{\prime}\right)+g_{k}\left(a_{k}^{\prime}\right) \quad \forall k \in \mathbb{N}
$$

в силу соотношений $(5.15),(5.18),(5.19)$ имеем

$$
\limsup _{k \rightarrow \infty}\left(\left(f_{k} \boxplus g_{k}\right)\left(x_{k}\right)-(f \boxplus g)\left(x_{k}\right)\right) \leqslant 0 .
$$

С другой стороны, по определению эписуммы имеют место неравенства

$$
(f \boxplus g)\left(x_{k}\right) \leqslant f\left(x_{k}-b_{k}^{\prime}\right)+g\left(b_{k}^{\prime}\right) \quad \forall k \in \mathbb{N} .
$$

Поэтому согласно соотношениям (5.16), (5.18), (5.19) получаем

$$
\limsup _{k \rightarrow \infty}\left((f \boxplus g)\left(x_{k}\right)-\left(f_{k} \boxplus g_{k}\right)\left(x_{k}\right)\right) \leqslant 0 .
$$

Отсюда и из соотношения (5.20) следует равенство (5.14), из которого вытекает доказываемое утверждение.

Лемма 5.5. Пусть $w \in \mathfrak{C} \mathfrak{B}(E), R>0, T>0, t>0$, последовательность функций $f_{k} \in \operatorname{WConv}\left(w_{[R]}\right) \cap \operatorname{WConc}\left(w_{[T]}\right)$ сходится в смысле $\mathfrak{E}$ $\kappa$ функции $f_{0} \in \operatorname{WConv}\left(w_{[R]}\right) \cap \operatorname{WConc}\left(w_{[T]}\right)$, а последовательность функций $g_{k} \in \operatorname{SConv}\left(w_{[t]}\right)$ сходится в смысле $\mathfrak{C} \mathfrak{B} \kappa$ функиии $g_{0} \in \operatorname{SConv}\left(w_{[t]}\right)$. Тогда:

(i) ecлu $t<R$, mo $f_{k} \boxplus g_{k} \underset{\mathfrak{C B}}{\longrightarrow} f_{0} \boxplus g_{0}$;

(ii) если $t<T$, mo $f_{k} \boxminus g_{k} \underset{\mathfrak{C} \mathfrak{B}}{\longrightarrow} f_{0} \boxminus g_{0}$.

ДоказАтельство. Утверждение (i) следует из леммы 5.4. Докажем утверждение (ii). Для любого индекса $k \in \mathbb{N} \cup\{0\}$ определим функцию $\widetilde{f}_{k}(x)=$ $-f_{k}(-x)$. Тогда $\widetilde{f}_{k} \in \operatorname{WConv}\left(w_{[T]}\right) \cap \operatorname{WConc}\left(w_{[R]}\right),\left(f_{k} \boxminus g_{k}\right)(x)=-\left(\widetilde{f}_{k} \boxplus g_{k}\right)(-x)$ для любого $k \in \mathbb{N} \cup\{0\}$. Применяя (i), получаем (ii). Лемма доказана.

ТЕОРЕма 5.1 (непрерывная зависимость седловой точки от параметров). Пусть $w \in \mathfrak{C} \mathfrak{B}(E), 0<r<R, 0<t<T$, последовательность функций $f_{k} \in \operatorname{WConv}\left(w_{[R]}\right) \cap \operatorname{WConc}\left(w_{[T]}\right)$ сходится в смысле $\mathfrak{C} \mathfrak{B} \kappa$ функиии $f_{0} \in \mathrm{WConv}\left(w_{[R]}\right) \cap \mathrm{WConc}\left(w_{[T]}\right)$. Пусть последовательность $g_{k} \in \operatorname{SConv}\left(w_{[r]}\right)$ сходится в смысле $\mathfrak{C} \mathfrak{B} \kappa$ функиии $g_{0} \in \operatorname{SConv}\left(w_{[r]}\right)$ и последовательность функций $h_{k} \in \operatorname{SConv}\left(w_{[t]}\right)$ сходится в смысле $\mathfrak{C} \mathfrak{B} \kappa$ функиии $h_{0} \in \operatorname{SConv}\left(w_{[t]}\right)$. Пусть последовательность векторов $x_{k} \in E$ сходится по норме $E \kappa$ вектору $x_{0} \in E$ и для любого индекса $k \in \mathbb{N} \cup\{0\}$ пара векторов $\left(u_{k}, v_{k}\right) \in E \times E$ составляет седловую точку функиии

$$
F_{k}(u, v)=f_{k}\left(x_{k}-u+v\right)+g_{k}(u)-h_{k}(v) .
$$

Тогда последовательности $\left\{u_{k}\right\}$ u $\left\{v_{k}\right\}$ сходятся по норме $Е$ соответственно $\kappa$ векторам $u_{0} u v_{0}$.

ДоказАтельство. Для любого индекса $k \in \mathbb{N} \cap\{0\}$ определим функцию $H_{k}=f_{k} \boxminus h_{k}$. По определению седловой точки имеем

$$
H_{k}\left(x_{k}-u_{k}\right)+g_{k}\left(u_{k}\right)=\left(H_{k} \boxplus g_{k}\right)\left(x_{k}\right) \quad \forall k \in \mathbb{N} \cap\{0\} .
$$


В силу леммы 5.5,(ii) справедливо соотношение $H_{k} \underset{\mathfrak{C B}}{\longrightarrow} H_{0}$. Применяя лемму 5.3,(ii), получаем, что $\lim _{k \rightarrow \infty}\left\|u_{k}-u_{0}\right\|=0$. Применяя полученный результат для функций

$$
\tilde{f}_{k}(x)=-f_{k}(-x), \quad \widetilde{g}_{k}=h_{k}, \quad \widetilde{h}_{k}=g_{k}, \quad \widetilde{F}_{k}(v, u)=-F_{k}(u, v),
$$

имеем соотношение $\lim _{k \rightarrow \infty}\left\|v_{k}-v_{0}\right\|=0$. Теорема доказана.

\section{§ 6. Применение теоремы о седловой точке в теории дифференциальных игр}

Теория антагонистических дифференциальных игр рассматривает задачи управления динамической системой двумя игроками, имеющими противоположные цели. В рамках теории дифференциальных игр может быть исследовано оптимальное управление объектом в конфликтных ситуациях, а также в ситуациях, когда на объект воздействует помеха, играющая роль одного из игроков. Задача состоит в нахождении оптимальной стратегии управления объектом, обеспечивающей оптимальный гарантированный результат, т. е. наилучший результат (значение целевого функционала), который может достичь игрок при самых неблагоприятных действиях соперника.

Большинство современных алгоритмов построения стратегий в дифференциальных играх используют метод стабильного моста Н. Н. Красовского [9] или альтернированный интеграл Л. С. Понтрягина [10]. Как показали многочисленные работы, эти алгоритмы весьма трудоемки и не всегда могут быть реализованы на практике. Ситуация значительно упрощается, если в дифференциальной игре существует седловая точка в классе программных стратегий. В этом случае оптимальную стратегию можно находить с помощью более простых алгоритмов, основанных на принципе максимума Л. С. Понтрягина. В этом параграфе получены достаточные условия, при которых для дифференциальной игры седловая точка в классе программных стратегий существует.

Рассмотрим линейную дифференциальную игру с динамикой

$$
\frac{d x}{d t}=B(t) v(t)-A(t) u(t), \quad t \in[0, \vartheta],
$$

начальным условием

$$
x(0)=x_{0}
$$

и целевым функционалом

$$
J\left(x_{0}, u, v\right)=f(x(\vartheta))+\int_{0}^{\vartheta}(\alpha(t, u(t))-\beta(t, v(t))) d t .
$$

Здесь $u:[0, \vartheta] \rightarrow \mathbb{R}^{p}, v:[0, \vartheta] \rightarrow \mathbb{R}^{q}$ - управления игроков, $x(t) \in \mathbb{R}^{n}$ - фазовый вектор системы, $A:[0, \vartheta] \rightarrow \mathbb{R}^{n \times p}, B:[0, \vartheta] \rightarrow \mathbb{R}^{n \times q}$ - заданные кусочно непрерывные матричнозначные функции.

Управления игроков подчиняются геометрическим ограничениям

$$
u(t) \in P(t), \quad v(t) \in Q(t) \quad \forall t \in[0, \vartheta],
$$


где $P, Q$ - заданные многозначные отображения, значениями которых являются выпуклые множества $P(t) \subset \mathbb{R}^{p}, Q(t) \subset \mathbb{R}^{q}$.

Цель игрока $u$ состоит в минимизации функционала $J$, цель игрока $v-$ в его максимизации.

Заметим, что дифференциальная игра с линейной динамикой

$$
\frac{d x}{d t}=C(t) x(t)+B(t) v(t)-A(t) u(t), \quad t \in[0, \vartheta],
$$

линейным преобразованием фазового вектора системы сводится к игре с динамикой (6.1). Поэтому полученные здесь результаты применимы к дифференциальным играм с динамикой (6.5).

Функцию $f: \mathbb{R}^{n} \rightarrow \mathbb{R}$, определяющую зависимость целевого функционала (6.3) от значения фазового вектора в конечный момент времени $\vartheta$, будем называть терминальной. Функции $\alpha, \beta$ будем называть штрафными.

Будем предполагать, что штрафные функции $\alpha:[0, \vartheta] \times \mathbb{R}^{p} \rightarrow \mathbb{R} \cup\{+\infty\}$ и $\beta:[0, \vartheta] \times \mathbb{R}^{q} \rightarrow \mathbb{R} \cup\{+\infty\}$ являются борелевскими и согласованы с множествами допустимых управлений игроков (6.4), а именно

$$
\operatorname{dom} \alpha(t, \cdot)=P(t), \quad \operatorname{dom} \beta(t, \cdot)=Q(t) \quad \forall t \in[0, \vartheta] .
$$

Кроме того, для любого $t \in[0, \vartheta]$ функции $u \mapsto \alpha(t, u)$ и $v \mapsto \beta(t, v)$ выпуклы и полунепрерывны снизу. Пусть существуют интегрируемые по Лебегу функции $\alpha_{0}:[0, \vartheta] \rightarrow \mathbb{R}, \beta_{0}:[0, \vartheta] \rightarrow \mathbb{R}$ и коэрцитивная функция $\varphi:[0,+\infty) \rightarrow[0,+\infty)$ такие, что

$$
\begin{gathered}
\alpha(t, u) \geqslant \alpha_{0}(t)+\varphi(\|u\|), \quad \beta(t, v) \geqslant \beta_{0}(t)+\varphi(\|v\|) \\
\forall t \in[0, \vartheta], \quad \forall u \in \mathbb{R}^{p}, \quad \forall v \in \mathbb{R}^{q} .
\end{gathered}
$$

Множеством $\mathcal{U}$ допустимых программных стратегий игрока и будем называть множество интегрируемых по Лебегу функций $u:[0, \vartheta] \rightarrow \mathbb{R}^{p}$ таких, что $u(t) \in P(t)$ для любых $t \in[0, \vartheta]$ и $\int_{0}^{\vartheta} \alpha(t, u(t)) d t<+\infty$. Множеством $\mathcal{V}$ допустимых программных стратегий игрока $v$ будем называть множество интегрируемых по Лебегу функций $v:[0, \vartheta] \rightarrow \mathbb{R}^{q}$ таких, что $v(t) \in Q(t)$ для любых $t \in[0, \vartheta]$ и $\int_{0}^{\vartheta} \beta(t, v(t)) d t<+\infty$.

Для любых векторов $\widetilde{u}, \widetilde{v} \in \mathbb{R}^{n}$ определим интегральные штрафные функции

$$
g(\widetilde{u})=\inf _{\substack{u \in \mathcal{U}: \\ \int_{0}^{\vartheta} A(t) u(t) d t=\widetilde{u}}} \int_{0}^{\vartheta} \alpha(t, u(t)) d t, \quad h(\widetilde{v})=\inf _{\substack{v \in \mathcal{V}: \\ \int_{0}^{\vartheta} B(t) v(t) d t=\widetilde{v}}} \int_{0}^{\vartheta} \beta(t, v(t)) d t .
$$

ЗАМЕчАнИЕ 6.1. При выполнении перечисленных выше предположений согласно [11, гл. VIII, теорема 2.2] для любых $\widetilde{u} \in \operatorname{dom} g, \widetilde{v} \in \operatorname{dom} h$ инфимумы (6.6) достигаются.

ТЕОРема 6.1. Пусть выполнены предположения, принятые в настоящем nараграфе. Пусть заданы функиия $w \in \mathfrak{C} \mathfrak{B}\left(\mathbb{R}^{n}\right)$ и числа $r, t, R, T$ такие, что 
$R>r>0, T>t>0$. Пусть терминальная функиия $f$ и интегральные штрафные функиии $g, h$ удовлетворяют условиям

$$
f \in \operatorname{WConv}\left(w_{[R]}\right) \cap \operatorname{WConc}\left(w_{[T]}\right), \quad g \in \operatorname{SConv}\left(w_{[r]}\right), \quad h \in \operatorname{SConv}\left(w_{[t]}\right) .
$$

Тогда для дифференциальной игрь (6.1)-(6.4) при любой началъной позиции (6.2) существует седловая точка в классе программных стратегий.

ДокАЗАТЕЛьство. Зафиксируем произвольный вектор $x_{0} \in \mathbb{R}^{n}$. В силу теоремы 1.1 существуют векторы $\widetilde{u}_{*} \in \mathbb{R}^{n}, \widetilde{v}_{*} \in \mathbb{R}^{n}$ такие, что

$$
f\left(x_{0}-\widetilde{u}_{*}+\widetilde{v}\right)+g\left(\widetilde{u}_{*}\right)-h(\widetilde{v}) \leqslant f\left(x_{0}-\widetilde{u}+\widetilde{v}_{*}\right)+g(\widetilde{u})-h\left(\widetilde{v}_{*}\right) \quad \forall \widetilde{u}, \widetilde{v} \in \mathbb{R}^{n} .
$$

В силу замечания 6.1 существуют функции $u_{*} \in \mathcal{U}, v_{*} \in \mathcal{V}$ такие, что

$$
\begin{array}{cc}
\int_{0}^{\vartheta} A(t) u_{*}(t) d t=\widetilde{u}_{*}, & \int_{0}^{\vartheta} B(t) v_{*}(t) d t=\widetilde{v}_{*}, \\
g\left(\widetilde{u}_{*}\right)=\int_{0}^{\vartheta} \alpha\left(t, u_{*}(t)\right) d t, & h\left(\widetilde{v}_{*}\right)=\int_{0}^{\vartheta} \beta\left(t, v_{*}(t)\right) d t .
\end{array}
$$

Пусть заданы произвольные программные стратегии $u \in \mathcal{U}, v \in \mathcal{V}$. Обозначим

$$
\widetilde{u}=\int_{0}^{\vartheta} A(t) u(t) d t, \quad \widetilde{v}=\int_{0}^{\vartheta} B(t) v(t) d t .
$$

Из равенств (6.6) следуют неравенства

$$
g(\widetilde{u}) \leqslant \int_{0}^{\vartheta} \alpha(t, u(t)) d t, \quad h(\widetilde{v}) \leqslant \int_{0}^{\vartheta} \beta(t, v(t)) d t .
$$

Отсюда и из неравенства (6.7) получаем

$$
\begin{aligned}
f\left(x_{0}\right. & \left.+\int_{0}^{\vartheta}\left(B(t) v(t)-A(t) u_{*}(t)\right) d t\right)+\int_{0}^{\vartheta}\left(\alpha\left(t, u_{*}(t)\right)-\beta(t, v(t))\right) d t \\
& \leqslant f\left(x_{0}+\int_{0}^{\vartheta}\left(B(t) v_{*}(t)-A(t) u(t)\right) d t\right)+\int_{0}^{\vartheta}\left(\alpha(t, u(t))-\beta\left(t, v_{*}(t)\right)\right) d t .
\end{aligned}
$$

Согласно соотношениям (6.1)-(6.3) последнее неравенство можно переписать в виде $J\left(x_{0}, u_{*}, v\right) \leqslant J\left(x_{0}, u, v_{*}\right)$. Отсюда в силу произвольности программных стратегий $u \in \mathcal{U}, v \in \mathcal{V}$ следует, что пара программных стратегий $\left(u_{*}, v_{*}\right)$ составляет седловую точку в дифференциальной игре (6.1)-(6.4). Теорема доказана.

\section{Список литературы}

1. Е. С. Половинкин, М. В. Балашов, Элементы выпуклого и сильно выпуклого анализа, 1-е изд., Физматлит, М., 2004; 2-е изд., испр и доп.; Физматлит, М., 2007.

2. Г. Е. Иванов, Слабо выпуклые множества и функиии, Физматлит, М., 2006. 
3. Б. Т. Поляк, "Теоремы существования и сходимости минимизирующих последовательностей для задач на экстремум при наличии ограничений", Докл. АH CCCP, 166:2 (1966), 287-290; англ. пер.: В. Т. Poljak, "Existence theorems and convergence of minimizing sequences in extremum problems with restrictions", Soviet Math. Dokl., 7 (1966), 72-75.

4. Дж. фон Нейман, "К теории стратегических игр", Теория игр. Матричные игры, Физматгиз, М., 1961, 173-204; пер. с англ.: J. von Neumann, "On the theory of games of strategy", Contributions to the theory of games, v. IV, Princeton Univ. Press, Princeton, NJ, 1959, 13-42.

5. K. Fan, "Fixed-point and minimax theorems in locally convex topological linear spaces", Proc. Nat. Acad. Sci. USA, 38:2 (1952), 121-126.

6. M. Sion, "On general minimax theorems", Pacific J. Math., 8 (1958), 171-176.

7. J.-J. Moreau, "Inf-convolution des fonctions numeriques sur un espace vectoriel", C. R. Acad. Sci. Paris, 256 (1963), 5047-5049.

8. Е. С. Половинкин, "О сильно выпуклых множествах и сильно выпуклых функциях", Тр. международной конферениии, посвященной 90-летию со дня рождения Л. С. Понтрягина (Москва, 1998), т. 2, Итоги науки и техн. Сер. Соврем. мат. и ее прил. Темат. обз., 61, Негладкий анализ и оптимизация, ВИНИТИ, М., 1999, 66-138; англ. пер.: E. S. Polovinkin, "On strongly convex sets and strongly convex functions", J. Math. Sci. (New York), 100:6 (2000), 2633-2681.

9. Н. Н. Красовский, Управление динамической системой, Наука, М., 1985.

10. Л. С. Понтрягин, “Линейные дифференциальные игры преследования", Матем. сб., 112(154):3(7) (1980), 307-330; англ. пер.: L. S. Pontrjagin, "Linear differential games of pursuit", Math. USSR-Sb., 40:3 (1981), 285-303.

11. И. Экланд, Р. Темам, Выпуклый анализ и вариачионные проблемы, Мир, М., 1979; пер. с англ.: I. Ekeland, R. Temam, Convex analysis and variational problems, Studies in Mathematics and its Applications, 1, North-Holland, Amsterdam-Oxford; Elsevier, New York, 1976.

Г. Е. Иванов (G. E. Ivanov)

Поступило в редакцию

Московский физико-технический институт

(государственный университет)

19.06.2009

E-mail: givanov@mail.mipt.ru 